\title{
Prediction of Sinorhizobium meliloti sRNA genes and experimental detection in strain $20 \mathrm{II}$
}

\author{
Claudio Valverde*1, Jonathan Livny ${ }^{2}$, Jan-Philip Schlüter ${ }^{3,4}$, \\ Jan Reinkensmeier ${ }^{5}$, Anke Becker ${ }^{3,4}$ and Gustavo Parisi ${ }^{6}$
}

\begin{abstract}
Address: ${ }^{1}$ Programa Interacciones Biológicas, Departamento de Ciencia y Tecnología, Universidad Nacional de Quilmes, Roque Sáenz Peña 352 , Bernal, Buenos Aires, B1876BXD, Argentina, ²Channing Laboratories, Brigham and Women's Hospital, Harvard Medical School, 181 Longwood Avenue, Boston, MA 02115, USA, ${ }^{3}$ Institute for Genome Research and Systems Biology, Center for Biotechnology (CeBiTec), Bielefeld University, 33594 Bielefeld, Germany, ${ }^{4}$ Institute of Biology III, Faculty of Biology, University of Freiburg, Schänzlestr. 1, 79104 Freiburg, Germany, ${ }^{5}$ Faculty of Technology, Bielefeld University, 33615 Bielefeld, Germany and ${ }^{6}$ Grupo de Bioinformática Estructural, Centro de Estudios e Investigaciones, Universidad Nacional de Quilmes, Roque Saénz Peña 352, Bernal, Buenos Aires, B1876BXD, Argentina
\end{abstract}

Email: Claudio Valverde* - cvalver@unq.edu.ar; Jonathan Livny - jlivny@rics.bwh.harvard.edu; Jan-Philip Schlüter - jschluet@Genetik.UniBielefeld.DE; Jan Reinkensmeier - jreinken@techfak.uni-bielefeld.de; Anke Becker - Anke.Becker@ Genetik.Uni-Bielefeld.DE;

Gustavo Parisi - gparisi_1@ @hotmail.com

* Corresponding author

Published: 16 September 2008

BMC Genomics 2008, 9:416 doi:10.1/86/147|-2164-9-416
Received: 4 January 2008

Accepted: 16 September 2008

This article is available from: http://www.biomedcentral.com/I47I-2/64/9/4I6

(c) 2008 Valverde et al; licensee BioMed Central Ltd.

This is an Open Access article distributed under the terms of the Creative Commons Attribution License (http://creativecommons.org/licenses/by/2.0), which permits unrestricted use, distribution, and reproduction in any medium, provided the original work is properly cited.

\begin{abstract}
Background: Small non-coding RNAs (sRNAs) have emerged as ubiquitous regulatory elements in bacteria and other life domains. However, few sRNAs have been identified outside several well-studied species of gammaproteobacteria and thus relatively little is known about the role of RNA-mediated regulation in most other bacterial genera. Here we have conducted a computational prediction of putative sRNA genes in intergenic regions (IgRs) of the symbiotic $\alpha$-proteobacterium S. meliloti 1021 and experimentally confirmed the expression of dozens of these candidate loci in the closely related strain S. meliloti $201 \mathrm{I}$.

Results: Our first sRNA candidate compilation was based mainly on the output of the sRNAPredictHT algorithm. A thorough manual sequence analysis of the curated list rendered an initial set of 18 lgRs of interest, from which 14 candidates were detected in strain $201 \mathrm{I}$ by Northern blot and/or microarray analysis. Interestingly, the intracellular transcript levels varied in response to various stress conditions. We developed an alternative computational method to more sensitively predict sRNA-encoding genes and score these predicted genes based on several features to allow identification of the strongest candidates. With this novel strategy, we predicted 60 chromosomal independent transcriptional units that, according to our annotation, represent strong candidates for sRNA-encoding genes, including most of the sRNAs experimentally verified in this work and in two other contemporary studies. Additionally, we predicted numerous candidate sRNA genes encoded in megaplasmids PSymA and pSymB. A significant proportion of the chromosomal- and megaplasmid-borne putative sRNA genes were validated by microarray analysis in strain $201 \mathrm{l}$.

Conclusion: Our data extend the number of experimentally detected S. meliloti sRNAs and significantly expand the list of putative sRNA-encoding lgRs in this and closely related $\alpha$-proteobacteria. In addition, we have developed a computational method that proved useful to predict sRNA-encoding genes in S. meliloti. We anticipate that this predictive approach can be flexibly implemented in many other bacterial species.
\end{abstract}




\section{Background}

In bacteria, small, non-coding RNA molecules that influence the expression of other genes are collectively referred to as sRNAs [1]. Significant experimental and theoretical evidence suggests sRNA-based regulation of gene expression is a paradigm common to all domains of life $[2,3]$. To date, two main mechanisms of sRNA activity have been described, both of which result in a modification of target mRNA translation and/or stability. The most common mechanism involves antisense pairing between the regulatory sRNA and the mRNA target [4]. In some cases, a single sRNA can mediate disparate regulatory effects on different mRNA targets. For instance, binding of the E. coli RyhB to the 5'-untranslated region of shiA mRNA activates shiA translation [5] whereas RhyB binding to sodB mRNA promotes its degradation [6]. In many cases the sRNA:mRNA interaction occurs over short regions of imperfect sequence complementarity and thus requires stabilization by the RNA chaperone Hfq [7]. The second sRNA-based mechanism is molecular mimicry, in which sRNAs offer multiple binding sites to RNA binding proteins of the CsrA/RsmA family, thus competitively relieving protein-mediated regulation of target mRNAs [8]. Most sRNAs characterized to date act as intermediate genetic elements of signal transduction cascades that are themselves initiated by a variety of external stimuli [9].

The number of putative and physically confirmed prokaryotic sRNAs has grown significantly in recent years, due in large part to the development and utilization of computational methods for predicting sRNA-encoding loci $[10,11]$. The pioneering predictive studies were initiated a few years ago when several groups discovered dozens of sRNAs in the intergenic regions of E. coli [12-14]. In these seminal studies, putative sRNAs were identified based on their association with genetic features common to several previously known sRNAs [15], such as their transcription from DNA regions between protein coding genes, their association with Rho-independent transcriptional terminator and/or promoter signals, the conservation of their primary sequence among closely related species, and their potential for encoding conserved secondary structure [16].

Sinorhizobium meliloti is an $\alpha$-proteobacterium able to establish an intimate symbiosis with the roots of legumes belonging to the genera Medicago, Melilotus and Trigonella [17]. Upon an intricate chemical dialog and cross-recognition between bacterium and roots, $S$. meliloti colonizes the interior of de novo root organs, the nodules, in which it differentiates into bacteroids committed to biological fixation of atmospheric nitrogen [18]. The genome of the sequenced strain $S$. meliloti 1021 is organized into three replicons, the "chromosome" $(3.65 \mathrm{Mb})$ and two megaplasmids, pSymA (1.35 Mb) and pSymB (1.68 Mb), that were most likely acquired through horizontally transfer. Sequence analysis indicates that pSymA, the giant plasmid devoted to nodulation and nitrogen fixation functions, was acquired later in the evolution of the host bacterium than pSymB [19-21]. The chromosome of S. meliloti encodes an $h f q$ homolog, suggesting that it also encodes sRNAs. However, prior to the initiation of this study, no screens for sRNAs had been conducted in Sinorhizobium and only the conserved chromosomal tmRNA homolog (ssrA) and an antisense countertranscript involved in control of pSymA and pSymB replication had been functionally characterized in S. meliloti [22-25]. While this work was in preparation, two groups reported the identification of a total of 15 chromosomally encoded sRNAs (including the widely conserved 6S RNA) and one pSymB-derived sRNA in $S$. meliloti strain $1021[26,27]$. These two studies employed similar predictive criteria, ones that were significantly different from the one utilized in this work. Here we report the prediction of dozens of putative sRNA genes encoded in the three replicons of $S$. meliloti and the experimental detection of many transcripts under different stress conditions in the closely related strain $S$. meliloti 2011. Our first sRNA candidate compilation was based mainly on the output of the sRNAPredictHT algorithm. A thorough manual sequence analysis of the curated list rendered an initial set of 18 IgRs of interest, from which 14 candidates were detected by Northern blot and/or microarray analysis. As we suspected that S. meliloti would encode more sRNA transcripts, we developed an alternative computational method to more sensitively predict sRNA-encoding genes, which introduces a novel cumulative scoring procedure to identify the strongest candidates. This scheme takes into account the location of predicted transcription signatures (promoters and terminators), their relative orientations and proximity to flanking protein coding genes, and their association with regions of conserved primary sequence and secondary structure. A novel scoring algorithm was integrated into this approach to allow the strongest candidate loci to be readily identified. Using this prediction and scoring approach we detected most of the $S$. meliloti small transcripts revealed by our first screening and in two recent studies [26,27] as well as numerous strong candidates for novel sRNAencoding genes in IgRs of $S$. meliloti chromosome and megaplasmids. A significant proportion of these chromosomal- and megaplasmid-borne putative sRNA genes were validated by microarray analysis.

\section{Methods}

First set of predicted sRNA-encoding genes in S. meliloti chromosomal intergenic regions

Among the 2920 chromosomal IgRs of S. meliloti 1021 [28], a first set of IgRs potentially encoding sRNAs (Table 1) was compiled by: 1) selection of IgRs with annotated orphan transcriptional terminators [28]; 2) selection of 
Table I: First compilation of S. meliloti chromosomal IgRs predicted to encode sRNAs.

\begin{tabular}{|c|c|c|c|c|c|c|c|c|c|c|c|}
\hline IgR\# & $\begin{array}{l}\text { Found } \\
\text { by' }\end{array}$ & $\begin{array}{l}\text { Adjacent } \\
\text { genes }^{2}\end{array}$ & $\begin{array}{l}\text { SRNA } \\
\text { strand }^{2}\end{array}$ & Prom.? ${ }^{3}$ & Term.? 3 & $\begin{array}{l}\text { Blast hits } \\
\text { in }^{4}\end{array}$ & $\begin{array}{l}\text { Expected } \\
\text { size }^{5}\end{array}$ & $\begin{array}{l}\text { Band size in } \\
\text { Northern blot }\end{array}$ & $\begin{array}{l}\text { Microarray } \\
\text { detection }^{7}\end{array}$ & Gene $^{8}$ & Reference \\
\hline I & Rfam & $\begin{array}{l}S M C 03975> \\
\text { SMc03976< }\end{array}$ & $<$ & s70 & $\mathrm{n}$ & eubacteria & $\sim 160 \mathrm{nt}$ & $\sim 155 \mathrm{nt}$ & $\begin{array}{c}<, H, C, S, A, B, \\
\text { O } \\
(2972187- \\
2972236)\end{array}$ & $\begin{array}{c}\text { 6S RNA } \\
(s m r C 22= \\
\text { sra56) }\end{array}$ & {$[26,27,62,67]$} \\
\hline 2 & OT & $\begin{array}{l}S M c 04042< \\
\text { SMc04043< }\end{array}$ & $>$ & $N N-s 70$ & $y$ & Sm RI Re At & $\sim 80 \mathrm{nt}$ & duplet $<100 \mathrm{nt}$ & - & $\operatorname{sm} 8=\operatorname{suh} B$ & $\begin{array}{c}{[65,67], \text { this }} \\
\text { work }\end{array}$ \\
\hline 3 & SP & $\begin{array}{c}S M c 01933< \\
\text { proS> }\end{array}$ & $<$ & $N N-s 70$ & $y$ & Sm Re RI At MI & $\sim 150 \mathrm{nt}$ & $\sim 165 \mathrm{nt}$ & $\begin{array}{c}<, A, C, H, S \\
(1398340- \\
1398389 \\
1398389- \\
1398340)\end{array}$ & $\operatorname{smrC9}=\operatorname{sra} 32$ & {$[26,27]$} \\
\hline 4 & $\mathrm{tR}$ & $\begin{array}{c}G \mid n-t R N A< \\
S M c 00810>\end{array}$ & $<$ & - & $y$ & Sm Re Rl & $?$ & $\begin{array}{c}\text { multiple } \sim 150 \\
\$ \sim \& 80 \mathrm{nt} \\
\text { mainly }\end{array}$ & - & $s m / 37$ & this work \\
\hline 5 & $\mathrm{tR}$ & $\begin{array}{c}S M c 02|5|< \\
\text { Thr-tRNA< }\end{array}$ & $>$ & $N N-s 70$ & y & - & $\sim 240 \mathrm{nt}$ & $\begin{array}{l}\text { no bands } \\
\text { detected }\end{array}$ & $\begin{array}{c}>, S(*) \\
(560802- \\
560753)\end{array}$ & sral2 & [27] \\
\hline 6 & OT & $\begin{array}{c}g l t B< \\
S M c 04029>\end{array}$ & $>$ & $\mathrm{NN}$ & y & Sm Re RI & $\sim 100-200 \mathrm{nt}$ & not done 6 & $\begin{array}{c}>, A, C \\
(3031662- \\
3031711)\end{array}$ & - & this work \\
\hline 7 & OT & $\begin{array}{l}S M c 04453< \\
\text { SMc0 I885> }\end{array}$ & $<$ & $\mathrm{NN}$ & $y$ & Sm Re Rl & $\sim 390 \mathrm{nt}$ & $\begin{array}{c}\text { duplet, } \sim 135 \& \\
\sim 200 \mathrm{nt}\end{array}$ & $\begin{array}{c}<, A, C, O, S \\
(2321429- \\
2321478)\end{array}$ & $\operatorname{sm} 26$ & this work \\
\hline 8 & OT & $\begin{array}{c}S M c 01257< \\
\text { sda }>\end{array}$ & $>$ & $\begin{array}{c}N N-s 70- \\
\text { PhoB }\end{array}$ & $y$ & Sm At Oa Msp & $\sim 170 \mathrm{nt}$ & not done 6 & - & $\operatorname{sm} 30$ & this work \\
\hline 9 & OT & thdF $<$ rho $<$ & $>$ & s70 & $y$ & Sm & $\sim 180 \mathrm{nt}$ & not done & - & $\mathrm{sm} / 30$ & this work \\
\hline 10 & SP & $\begin{array}{l}\text { SMc00034> } \\
\text { SMc00096< }\end{array}$ & $>$ & s70 & $y$ & Sm Re RI & $\sim 190 \mathrm{nt}$ & $\sim 175 \mathrm{nt}$ & - & sra25 & [27] \\
\hline
\end{tabular}




\begin{tabular}{|c|c|c|c|c|c|c|c|c|c|c|c|}
\hline II & $\mathrm{SP}$ & $\begin{array}{l}\text { SMc00108< } \\
\text { SMc00109< }\end{array}$ & $<$ & s70 & $y$ & Sm RI Re At & $\sim 100-120 \mathrm{nt}$ & $\begin{array}{l}\text { duplet, } \sim 120 \\
\$ \sim \& 110 \mathrm{nt}\end{array}$ & - & $s m / 45$ & this work \\
\hline 12 & $\mathrm{SP}$ & celR2 $>r p m G<$ & $>$ & s70 & $y$ & $\begin{array}{c}S m \operatorname{Rl} \operatorname{Re} A t M l \\
M s p A c\end{array}$ & $\sim 150 \mathrm{nt}$ & not done 6 & $\begin{array}{c}>\text {, A, B, C, H, O, } \\
\text { S } \\
(1411684- \\
1411733 \\
1411738- \\
1411689)\end{array}$ & $\begin{array}{c}\text { smrClo }= \\
\text { sra33 }\end{array}$ & {$[26,27]$} \\
\hline 13 & $\mathrm{SP}$ & $\begin{array}{c}\text { polA< } \\
\text { SMc0285I> }\end{array}$ & $>$ & $N N-s 70$ & $y$ & $S m R I R e A t$ & $\sim 150 \mathrm{nt}$ & $\begin{array}{c}\text { duplet, } \sim 140 \& \\
\sim 90 \mathrm{nt}\end{array}$ & - & smrC7 $=s r a 03$ & {$[26,27]$} \\
\hline 14 & $\mathrm{SP}$ & $\begin{array}{l}S M c 02910< \\
S M c 02911>\end{array}$ & $>$ & s70 & $y$ & $\begin{array}{c}S m \operatorname{Re} R I A t M l \\
\text { Bo Oa }\end{array}$ & $\sim 60-150 \mathrm{nt}$ & $\begin{array}{c}\text { multiple, } \sim 90- \\
100 \& \sim 180- \\
210\end{array}$ & $\begin{array}{c}\text { >, B, C, O, } \\
(267010-267059 \\
267059-267010)\end{array}$ & $s m 76$ & this work \\
\hline 15 & $\mathrm{SP}$ & $\begin{array}{l}\text { SMc03988> } \\
\text { SMc03989> }\end{array}$ & $>$ & s70 & y & Sm RI Re At & $\sim 110 \mathrm{nt}$ & $\sim 80 \mathrm{nt}$ & $\begin{array}{c}>, A, B, C, H, O, \\
\text { S } \\
(2986452- \\
2986501 \\
2986508- \\
2986459)\end{array}$ & sm84 & this work \\
\hline 16 & SP & $\begin{array}{c}a t p H< \\
\text { SMc02497< }\end{array}$ & $>$ & s70 & $y$ & Sm RI Re At & $\sim 110 \mathrm{nt}$ & $\sim 100 \mathrm{nt}$ & - & $s m 270$ & this work \\
\hline 17 & $\mathrm{SP}$ & $\operatorname{dapF}<f f h>$ & $>$ & s70 & $y$ & Sm RI Re At & $\sim 120 \mathrm{nt}$ & not done & $\begin{array}{c}>, A, B, O, S \\
(3522279- \\
3522328)\end{array}$ & $\operatorname{sm} 5$ & this work \\
\hline 18 & OT & $\begin{array}{l}S M c 00821> \\
\text { SMc00822> }\end{array}$ & $>$ & - & $y$ & Sm Re RI & $\sim 80 \mathrm{nt}$ & not done & $\begin{array}{l}>, \mathrm{A}, \mathrm{C}, \mathrm{H}, \mathrm{O}, \mathrm{S} \\
(843459-843508 \\
843508-843459)\end{array}$ & $\mathrm{sm} / 90$ & this work \\
\hline
\end{tabular}

I Rfam, RNA families database [33,67]; OT, IgR with annotated orphan terminator; tR, IgR flanking tRNAs; SP, sRNAPredictHT.

2 The transcribed strand is indicated (>, forward; <, reverse)

3 Putative promoter sequence detected by conservation in sequence alignment, search of consensus sequences or neural network promoter prediction. s70, sequence highly similar to the $S$. meliloti constitutive promoter consensus sequence [49]; NN, neural network promoter prediction [47]; PhoB, sequence detected with homology to the PhoB transcriptional regulator binding consensus [5I].

${ }^{4}$ Sm, Sinorhizobium medicae WSM4I9; RI, Rhizobium leguminosarum biovar viciae 384I; Re, Rhizobium etli CFN42; At, Agrobacterium tumefaciens C58; MI, Mesorhizobium loti MAFF303099; Oa,

Ochrobactrum anthropi ATCC49188; Msp, Mesorhizobium sp. BNCI; Bo, Brucella ovis ATCC25840.

${ }^{5}$ Approximate length of sRNA based on putative $5^{\prime}$ and $3^{\prime}$ ends.

${ }^{6} \mathrm{PCR}$ failed to amplify the IgR.

7 Detection on the oligonucleotide microarray Sm I4kOLI. $>$ and $<$ denote the orientation of the detected signal (absolute M-value $\geq 2.5$ represents an enrichment of small RNA transcripts). A,

$\mathrm{B}, \mathrm{C}, \mathrm{H}, \mathrm{O}$ and $\mathrm{S}$ denote the stress condition under which the signal was detected ( $\mathrm{A}$, acidic; $\mathrm{B}$, basic; $\mathrm{C}$, cold shock; $\mathrm{H}$, heat shock; $\mathrm{O}$, oxidative, $\mathrm{S}$, saline). The coordinates of the oligonucleotide probes that gave positive signals are indicated in brackets. -, not detected under the conditions studied. $(*), M$-value $\geq 2$.

${ }^{8}$ Gene name as designated in the literature (smrC\#, sra\#) [26,27]or according to our predictive scheme (sm\#). 
IgRs in the vicinity of tRNAs $[29,30] ; 3)$ application of sRNAPredictHT (J. Livny; unpublished data), an improved version of the program sRNApredict2 developed by Livny and co-workers [31]. Using default parameters, sRNAPredictHT identified 186 sequence elements as putative sRNAs (Additional file 1). However, almost 60\% of the hits corresponded to annotated $[19,28,32]$ or nonannotated sequence repeats. Each IgR was used to query Rfam database [33] to identify previously annotated RNA regulatory elements and then inspected for the presence of transcriptional signals (promoters and Rho-independent terminators; see below). We retained 17 chromosomal IgRs that were likely to encode sRNAs and an additional IgR encoding a putative 6S RNA homologue (Table 1).

Northern blot detection of the first set of sRNA candidates Sinorhizobium meliloti strain 2011 [34] was maintained on TY agar plates [35] with streptomycin $(400 \mu \mathrm{g} / \mathrm{ml})$. We chose the Rhizobium defined medium (RDM) [36] with shaking (120 rpm) at $28^{\circ} \mathrm{C}$ as the referential growth condition. For preparation of RNA extracts, $125-\mathrm{ml}$ flasks containing $20 \mathrm{ml}$ of RDM were inoculated with $0.2 \mathrm{ml}$ of a saturated RDM pre-culture and incubated at $120 \mathrm{rpm}$ until cell harvest. To introduce stress conditions, the RDM basal medium or growth conditions were modified as follows: high salt RDM $(0.3 \mathrm{M} \mathrm{NaCl})$, low phosphate RDM (0.1 mM phosphate, $10 \mathrm{mM}$ MOPS $\mathrm{pH} 7.0)$, RDM with ethanol $(2 \% \mathrm{v} / \mathrm{v}), \mathrm{RDM}$ with SDS $(0.1 \% \mathrm{w} / \mathrm{v})$ and RDM with $\mathrm{H}_{2} \mathrm{O}_{2}(0.1 \mathrm{mM})$. High temperature stress was applied by growing cells at $37^{\circ} \mathrm{C}$. For acid stress, exponential phase cells growing in $20 \mathrm{ml}$ of $\mathrm{RDM}\left(\mathrm{OD}_{600}=0.5\right)$ were collected by low speed centrifugation, washed with and resuspended in $20 \mathrm{ml}$ of RDM containing $20 \mathrm{mM}$ MES and equilibrated at $\mathrm{pH} 5.5$, and incubated $90 \mathrm{~min}$ at $28^{\circ} \mathrm{C}$ with shaking before harvesting cells for RNA extraction.

Total RNA was extracted immediately after cell harvest by low speed centrifugation $\left(1800 \mathrm{~g}, 10 \mathrm{~min}\right.$, and $\left.20^{\circ} \mathrm{C}\right)$. The cell pellet was resuspended in Trizol $^{\circledast}$ (Invitrogen; $1.5 \mathrm{ml}$ for cultures with $\mathrm{OD}_{600}<1.5$ or $3.0 \mathrm{ml}$ for cultures with $\mathrm{OD}_{600}>1.5$ ) and treated $1 \mathrm{~min}$ at $60^{\circ} \mathrm{C}$. Upon addition of 0.2 vols of chloroform and vigorous shaking during 15 secs, the RNA present in the aqueous supernatant was precipitated with $0.5 \mathrm{vol}$ of isopropanol. The pellet was washed in $70 \%$ ethanol, air dried and resuspended in 20 $\mu \mathrm{l}$ of DEPC-treated deionized water. RNA samples were conserved at $-130^{\circ} \mathrm{C}$. The purity and integrity of RNA preparations were assessed by denaturing PAGE electrophoresis followed by silver staining [37] and the RNA concentration was estimated by UV spectrometry [38]. For Northern blots, 1-3 $\mu \mathrm{g}$ RNA present in $1 \mu \mathrm{l}$ of each sample were fractionated on denaturing polyacrylamide gels $(60$ $\times 80 \times 0.75 \mathrm{~mm}$ containing $8.3 \mathrm{M}$ urea, $8 \%$ acrylamide and $0.2 \%$ bisacrylamide in $1 \times$ TBE buffer). The lane corresponding to the molecular weight markers (low range
RNA ladder; Fermentas) was cut out, stained with $5 \mu \mathrm{g} \mathrm{ml}-$ 1 ethidium bromide and photographed under UV light. The rest of the gel was electroblotted at $150 \mathrm{~mA}(15-25 \mathrm{~V})$ onto a Hybond-N membrane in $1 \times$ TBE buffer for $20 \mathrm{~min}$. Membranes were washed with $2 \times \operatorname{SSC}(0.3 \mathrm{M} \mathrm{NaCl}$ and 30 $\mathrm{mM}$ sodium citrate) before nucleic acids were cross-linked by exposure to UV light for $5 \mathrm{~min}$ [38]. Northern hybridizations were done with digoxigenin (DIG)-labeled DNA probes generated by PCR covering entirely or partially each IgR (Additional file 2). The IgR amplicons of detected candidate sRNAs were cloned in the $\mathrm{PCR}^{\circledR} 2.1$ TOPO vector and sequenced to confirm the identity of the PCR products. Hybridized membranes were developed following the protocol recommended by the manufacturer (Roche Diagnostics GmbH). The detected RNA bands were quantified by densitometry with ImageJ v1.38 [39] and standardized by the amount of loaded RNA visualized by silver staining.

\section{Microarray detection of sRNA candidates}

Pre-cultures of $S$. meliloti strain 2011 were grown at $30^{\circ} \mathrm{C}$ in TY [35] or GMS [40] media. For RNA isolation, $100 \mathrm{ml}$ flasks with $50 \mathrm{ml}$ TY or GMS medium, supplemented with $8 \mu \mathrm{g} / \mathrm{ml}$ nalidixic acid, were inoculated with $200 \mu \mathrm{l}$ of preculture and incubated in a rotary shaker $(175 \mathrm{rpm})$ at $30^{\circ} \mathrm{C}$ to an $\mathrm{OD}_{600}=0.6$. To induce stress, the medium and growth conditions were modified as follows. High salt stress: addition of $\mathrm{NaCl}$ to a final concentration of $0.4 \mathrm{M}$ in GMS medium. Oxidative stress: addition of $\mathrm{H}_{2} \mathrm{O}_{2}$ to a final concentration of $10 \mathrm{mM}$ in GMS medium. Cold shock stress: temperature shift of the culture in TY medium from $30^{\circ} \mathrm{C}$ to $20^{\circ} \mathrm{C}$. Heat shock stress: temperature shift of the culture in TY medium from $30^{\circ} \mathrm{C}$ to $40^{\circ} \mathrm{C}$. Acid or alkaline stress: cultures grown in GMS to an $\mathrm{OD}_{600}$ $=0.6$ were centrifuged and then re-suspended in GMS modified by adding $\mathrm{HCl}$ to $\mathrm{pH} 5.8$, or by adding $\mathrm{NaOH}$ to $\mathrm{pH}$ 8.5. In all cases, cells were harvested 15 and $45 \mathrm{~min}$ after exposure to stress conditions.

RNA was isolated and separated into small RNA $(<200$ $\mathrm{nt}$ ) and long RNA (> $200 \mathrm{nt}$ ) fractions using the miRNeasy Mini Kit (Qiagen) or Ambion mirVana miRNA Isolation Kit (Ambion) according to the manufacturers' instructions. Quality of RNA was analyzed applying the Agilent RNA 6000 Pico Kit on the Agilent 2100 Bioanalyzer (Agilent Technologies). To consider both orientations, aliquots from the same fractions of small and long RNA pools were sense labelled using the mirVana miRNA Labeling Kit (Ambion) and antisense labelled as described [41]. Differing from the cDNA labelling procedure [41], small RNA fractions were first tailed with PolyA polymerase (Ambion). Oligo dT and amino-allyl random hexamer primers were used for the synthesis of cDNA. 
Hybridization of the small RNA fraction (Cy3-fluorescent marker) was compared to that of the long RNA fraction (Cy5-fluorescent marker). Three combinations were performed: 1 . the small RNA fraction with the long RNA fraction, both of which were sense labelled, 2 . the same fractions in which both were antisense labelled, and 3. a combination of the sense labelled small RNA fraction and the antisense labelled long RNA fraction. Slide processing, sample hybridization, and scanning procedures were performed as described [41] applying the Sm14kOLI microarray that carries 50 mer to 70 mer oligonucleotide probed directed against coding regions and intergenic regions [42]. Analysis of microarray images was carried out applying the ImaGene 6.0 software (BioDiscoveries) [41]. Lowess normalization and significance test (fdr) were performed with the EMMA software [43]. The MValue represents the logarithmic ratio between both channels. The A-Value represents the logarithm of the combined intensities of both channels. Positive M-values $\geq 2.5$ represent an enrichment of small RNA fragments $(<=200$ nt) and therefore were classified as sRNA candidates.

\section{Novel method for in silico identification of sRNA candidate genes}

From the original 2920 chromosomal IgRs, all the annotated repetitive elements of 1021 chromosome (Smrepeats, RIMEs and $A B, C$ motifs) $[19,28,32]$ were removed and the flanking IgR segments were treated as new IgRs. 1720 chromosomal IgRs free of annotated repeats and longer than $150 \mathrm{nt}$ were retained for further analysis. Certain IgRs were also removed if they gave Blast $\mathrm{N}$ hits with E-value $<10^{-3}$ when queried against themselves, reducing the number of IgRs to 778 . With the help of open source algorithms and web based tools, the 778 chromosomal IgRs were subjected to the following sequence analyses: prediction of Rho-independent transcription terminators and of promoter signals, sequence conservation (BlastN; [44]) and secondary structure conservation (QRNA analysis) [45].

For prediction of Rho-independent transcription terminators, the web based TranstermHP server [46] was queried to generate a list of putative terminator sequences in chromosomal IgRs of strain 1021, having a stem length of 423 bases, a hairpin score $\leq-1.5$, a tail score $\leq-2.0$ and $\geq$ $80 \%$ of confidence. Orphan terminators (i.e., those that do not correspond to flanking CDS) were scored 3. Predicted terminators co-oriented with flanking ORFs were scored according to their relative distance to the 3'-end of the corresponding annotated gene so that a score of 2 was assigned if the terminator was farther than $200 \mathrm{bp}, 1$ if the distance was $100-200 \mathrm{nt}$, and 0 if it was closer than 100 bp.
Promoter signals were predicted with three alternative methods. A first set of putative promoters was generated with a web based neural network based routine [47] set up for bacterial sequences in both DNA strands with a minimum score of 0.8 . A second set of putative promoters was compiled by querying IgRs with Fuzznuc [48] using available $S$. meliloti consensus sequences as input. For $\sigma^{70}$ dependent promoters the query was CTT$\operatorname{GAC}\left(\mathrm{N}_{17}\right)$ CTATAT [49] with up to 4 mismatches allowed. For $\sigma^{54}$-dependent promoters the query was TGGCACG $\left(\mathrm{N}_{4}\right)$ TTGCW [50] with up to 2 mismatches allowed. For putative PhoB-binding sites the results of two queries were pooled, CTGTCAT( $\left.\mathrm{N}_{4}\right)$ CTGTCAT [51] with up to 4 mismatches allowed and TGWCAM $\left(\mathrm{N}_{4}\right)$ CYKTCAK [52] with up to 2 mismatches allowed. A third group of promoters was predicted with the help of the matrix-scan tool available at the Rsat web server [53], upon introduction of available scoring matrices for $S$. meliloti $\sigma^{70_{-},} \sigma^{54_{-}}$ and PhoB-dependent promoters [49-52] and with default parameters. A similar scoring criterion to that used for terminators was applied to predicted promoters. Orphan promoters were scored 3. Putative promoters were rated 2 if the 5 '-end of the co-oriented flanking CDS was farther than $300 \mathrm{bp}, 1$ if this distance was 200-300 bp and 0, if they were closer than $200 \mathrm{bp}$.

Similarity searches performed with BlastN were done using default parameter values. IgRs were used to query against a database of 559 complete eubacterial genomes [54] and we defined a Blast score (\#BlastN) that for each input IgR sequence consists in the sum of all the hits with E-values below $10^{-3}$. We used QRNA [45] to analyze the sequence alignments generated for each IgR and a score was derived summing all the positive hits detected (\#QRNA).

Finally, the individual scores for predicted terminators (\#T), promoters (\#P), BlastN (\#BlastN) and QRNA analysis (\#QRNA) were combined to generate a Global Score (GS). If a putative promoter and a terminator lay co-oriented and separated from each other by $40-500 \mathrm{bp}$, suggesting the presence of a single and independent transcriptional unit, the IgR is scored 10 and the individual scores for promoter and terminator are no longer considered. The GS for those IgRs containing such putative elements indicative of sRNAs was calculated as $(10+$ \#BlastN + \#QRNA). For those IgRs lacking putative independent transcriptional units, the GS was calculated as (\#T + \#P + \#BlastN + \#QRNA).

\section{Results \& Discussion \\ A first selection of chromosomal intergenic regions potentially encoding sRNAs}

At the time we initiated this study, the only chromosomal non-coding RNA gene that had been characterized in the 
$\alpha$-proteobacterium $S$. meliloti was the tmRNA homolog ssrA [23]. However, several findings suggested that other sRNAs might be expressed in this $\alpha$-proteobacterium. The electrophoretic fractionation in denaturing polyacrylamide gels of total RNA from strain 2011 cells grown under different conditions (Additional file 3) revealed several RNA bands of $<300 \mathrm{nt}$ other than the conserved and abundant 5S RNA, 4.5S RNA and tRNAs [27]. Another indirect evidence of the existence of sRNAs in S. meliloti comes from the pleiotropic phenotype of the $S$. meliloti $2011 \mathrm{hfq}$ mutant (Sobrero \& Valverde, unpublished). These observations suggest that the product of the $h f q$ gene $(\mathrm{SMc01048}=n r f A)$ may be required to assist diverse regulatory interactions between mRNAs and sRNAs, as reported for other bacterial species [7,55]. We thus decided to perform a bioinformatic search of sRNA genes using the genomic information of the sequenced strain $S$. meliloti 1021 .

Although there are reports of sRNAs transcribed from coding regions in other bacteria $[56,57]$, we focused our search in the regions between annotated ORFs (hereafter IgRs) of the S. meliloti chromosome [19]. We first identified in the $S$. meliloti annotated database [28] chromosomal IgRs containing transcriptional terminators unlikely to be associated with flanking ORFs as well as regions of sequence conservation in the vicinity of annotated tRNA genes, which may represent horizontally transferred genetic elements $[29,30]$. This "manual" procedure resulted in the identification of a few interesting IgRs (tagged OT and tR in Table 1). Next, we applied sRNAPredictHT, an improved version of the systematic and integrative tool sRNApredict 2 already used for the prediction of sRNA genes in several bacterial species [31]. sRNAPredictHT identifies sRNA-encoding loci based on the colocalization of transcriptional terminators and $\mathrm{IgR}$ sequence conservation [31]. Among the 186 candidate loci identified by sRNAPredictHT (Additional file 1), 56\% were identified in IgRs containing at least one repetitive DNA element, either the annotated Rhizobium-specific intergenic mosaic elements (RIMEs) [19,32], Sm-repeats $[19,28], A B, C$ palindromes $[19,28]$, or in some cases, even non-annotated repeats. Rhizobial genomes are characterized for the presence of dozens of these intergenic sequences of unknown function that typically share significant primary sequence and secondary structure conservation [58]. Upon elimination of IgRs containing repeats, the sRNAPredictHT output was narrowed down to a list of 76 candidate IgRs (Additional file 4). To further reduce the number of IgRs for experimental verification, we looked for candidates associated with putative promoters. This stringent filtering yielded a list of 17 interesting IgRs (Table 1). In fact, 15 candidate IgRs have both potential 5' and 3' transcriptional signals and are conserved in related species (Table 1), suggesting that they correspond to bona fide sRNA-encoding genes. Table 1 also includes a putative homolog of the widely conserved 6S RNA (IgR\#1; [33]) which was not picked up by sRNAPredictHT because it lacks a typical Rho-independent terminator (Table 1). With the exception of IgR\#5, all the candidates in Table 1 are conserved in at least one related $\alpha$-proteobacterium. All IgRs but the aforementioned IgR\#1 (6S RNA) are associated with a predicted Rho-independent terminator.

\section{Experimental verification of selected sRNA candidates in S. meliloti strain $201 \mathrm{I}$}

For experimental verification of most putative sRNA genes listed in Table 1, we performed Northern hybridizations and microarray analysis of RNA from $S$. meliloti strain 2011 that, like the sequenced strain 1021, is a streptomycin-resistant mutant derived from the isolate SU47. Although the separate and parallel continuous manipulation of these isogenic strains gave origin to subtle differences in their symbiotic behaviour and gene expression $[52,59]$, the overall high degree of sequence similarity between both strains permits the use of strain 2011 to test predictions based on 1021 sequence. As many characterized sRNAs are involved in regulatory processes induced by a variety of external stimuli [9], RNA extracts were prepared from cells grown both under standard culture conditions and under a variety of stressful conditions.

Of the 12 candidate IgRs from our initial compilation that were subjected to experimental verification by Northern analysis of S. meliloti 2011 RNA, 11 were detected (Table 1, Figure 1, Additional file 5). For the majority, the transcript size was consistent with our predictions (Table 1). In some cases (e.g., IgR\#10, IgR\#11 or IgR\#13), multiple bands were observed. Two IgRs (\#4 and \#14) revealed a complex banding pattern (Table 1; Additional file 5) and further experiments are required to elucidate the origin of the detected RNA bands. Microarray analysis of strain 2011 RNA detected enrichment of RNA molecules $<200$ nt corresponding to the predicted DNA regions for IgR\#1, IgR\#3, IgR\#6, IgR\#7, IgR\#12, IgR\#14, IgR\#15, IgR\#17 and IgR\#18 (M-value > 2.5; Table 1). For the rest of the IgRs for which no signals were detected in Northern blot or microarray analysis, it may be that the transcript level is below our threshold of detection or that this candidate sRNA has a very specific inducing signal different from those included in our assays. This may be the case for IgR\#5 with no detected bands in Northern blot (Figure 1) and a slightly lower enrichment detected in the microarray experiment $(\mathrm{M}$-value $=2.15$ under $45 \mathrm{~min}$ of saline stress; Table 2). In fact, two transcripts of different polarity (sra12a and sra12b) were reported for the same IgR in strain 1021 [27]. During the preparation of this manuscript, transcripts were reported in total RNA from strain 1021 for IgR\#1, IgR\#3, IgR\#5, IgR\#10, IgR\#12 and IgR\#13 $[26,27]$. Thus, our data independently confirmed the 
Table 2: Top 20 highest-scoring putative sRNA genes predicted by the global scoring procedure as independent transcriptional units in chromosomal IgRs of $S$. meliloti I02I.

\begin{tabular}{|c|c|c|c|c|c|c|c|c|c|c|c|c|c|c|}
\hline $\begin{array}{l}\text { Gene or } \\
\text { Designation }\end{array}$ & $\begin{array}{c}\text { IgR } \\
\text { length }\end{array}$ & $\begin{array}{l}\text { Upstream } \\
\text { ORF }\end{array}$ & & nta & & Promoter $^{2}$ & $\begin{array}{l}\text { Predicted } \\
5^{\prime} \text {-end }{ }^{3}\end{array}$ & $\begin{array}{l}\text { Predicted } \\
3^{\prime} \text {-end }{ }^{4}\end{array}$ & $\begin{array}{l}\text { Blast } \\
\text { score }\end{array}$ & $\begin{array}{l}\text { Qrna } \\
\text { score }\end{array}$ & $\begin{array}{l}\text { Global } \\
\text { score }\end{array}$ & $\begin{array}{l}\text { Length } \\
(\mathrm{nt})^{5}\end{array}$ & SP6 & $\begin{array}{l}\text { Microarray } \\
\text { detection }^{7}\end{array}$ \\
\hline $\begin{array}{l}\text { smrCl5 } \\
(s m 3) \\
\text { smrCl6 } \\
(s m 3 ')\end{array}$ & 652 & SMc0I 226 & $\begin{array}{l}< \\
<\end{array}$ & $\begin{array}{l}< \\
<\end{array}$ & $\begin{array}{l}< \\
<\end{array}$ & $\begin{array}{l}\text { NN, s70 } \\
\text { PhoB }\end{array}$ & $\begin{array}{c}1698732 \\
1698968 *\end{array}$ & $\begin{array}{l}1698618 \\
1698820\end{array}$ & 6 & 2 & 98 & $\begin{array}{l}114 \\
151\end{array}$ & y & $\begin{array}{c}<, C \\
(1698954- \\
1698905)\end{array}$ \\
\hline sm4 & 541 & SMc0I844 & $>$ & $>$ & $<$ & $\mathrm{NN}$, s70 & $\begin{array}{l}2371490 \\
2371606\end{array}$ & 2371852 & I & I & 82 & $256-362$ & $\mathrm{n}$ & $\begin{array}{c}>, S \\
(2371745- \\
2371696 \\
2371735- \\
2371784)\end{array}$ \\
\hline sm5 & 384 & SMc03856 & $<$ & $>$ & $>$ & s70 & $\begin{array}{l}3522121 \\
3522271\end{array}$ & 3522379 & 12 & 8 & 80 & $108-258$ & y & $\begin{array}{c}>, A, B, O, S \\
(3522279- \\
3522328)\end{array}$ \\
\hline sm6 & 491 & SMc0I202 & $<$ & $<$ & $<$ & $\mathrm{NN}, \mathrm{s} 70$ & $\begin{array}{l}1728153 \\
1728196 \\
1728269\end{array}$ & $|72802|$ & I & 1 & 62 & $120-248$ & $\mathrm{n}$ & - \\
\hline $\begin{array}{l}\text { smrCl4 } \\
\text { (sm7) } \\
\text { sm7' }\end{array}$ & 922 & SMc0205I & $\begin{array}{l}< \\
<\end{array}$ & $<$ & $\begin{array}{l}< \\
<\end{array}$ & $\begin{array}{c}\text { NN, s70 } \\
\text { NN }\end{array}$ & $\begin{array}{l}16676 \mid 4 \\
1667983\end{array}$ & $\begin{array}{l}1667488 \\
1667769\end{array}$ & 3 & 5 & 58 & $\begin{array}{l}126 \\
214\end{array}$ & $\mathrm{n}$ & $\begin{array}{c}<, S \\
(1667516- \\
1667467)\end{array}$ \\
\hline sm8 & 301 & SMc04042 & $<$ & $>$ & $<$ & $\mathrm{NN}$, s70 & 3046713 & 3046789 & 9 & 4 & 53 & 76 & y & - \\
\hline $\operatorname{sm} 9$ & 470 & SMc02080 & $>$ & $<$ & $>$ & $\mathrm{NN}$, s70 & $\begin{array}{l}\text { 1635343, } \\
\text { I6354II, } \\
1635564\end{array}$ & 1635217 & I & I & 52 & $126-347$ & $\mathrm{n}$ & $\begin{array}{c}<, \mathrm{B}, \mathrm{H}, \mathrm{O}\left(^{*}\right) \\
(1635305- \\
1635354 \\
1635259- \\
1635210)\end{array}$ \\
\hline $\mathrm{sm} / 0$ & 405 & SMc00057 & $>$ & $>$ & $>$ & $\mathrm{NN}, \mathrm{s} 70$ & $\begin{array}{l}1091047 \\
1091106\end{array}$ & 1091343 & 1 & 1 & 52 & $237-296$ & $n$ & - \\
\hline $\begin{array}{l}\text { smrC9 } \\
(s m / 2)\end{array}$ & 710 & SMc01933 & $<$ & $<$ & $>$ & $\mathrm{NN}$, s70 & $\begin{array}{l}\text { I398427, } \\
1398584\end{array}$ & 1398279 & 4 & 3 & 47 & $148-305$ & y & $\begin{array}{c}<, \mathrm{A}, \mathrm{C}, \mathrm{H}, \mathrm{S} \\
(1398340- \\
1398389 \\
1398389- \\
1398340)\end{array}$ \\
\hline
\end{tabular}


Table 2: Top 20 highest-scoring putative sRNA genes predicted by the global scoring procedure as independent transcriptional units in chromosomal IgRs of S. meliloti I02I.

\begin{tabular}{|c|c|c|c|c|c|c|c|c|c|c|c|c|c|c|}
\hline $\begin{array}{l}\text { smrC7 } \\
(\mathrm{sm} / 3)\end{array}$ & 472 & SMc02850 & $<$ & $>$ & $>$ & $\mathrm{NN}, \mathrm{s70}$ & 201682 & 201829 & 4 & 3 & 47 & 147 & $y$ & - \\
\hline$s m / I$ & 1101 & SMc0I67I & $<$ & $>$ & $<$ & NN, s70 & $\begin{array}{l}2475717 \\
2475862\end{array}$ & $247596 I$ & 5 & 2 & 47 & $99-244$ & y & - \\
\hline$s m / 4$ & 410 & SMc02I39 & $<$ & $>$ & $>$ & NN, PhoB & $\begin{array}{l}573816 \\
573830 *\end{array}$ & 574040 & 4 & 1 & 45 & 210 & $y$ & - \\
\hline $\begin{array}{l}\text { sral2 } \\
(\operatorname{sm} \mid 7)\end{array}$ & 829 & SMc02I5I & $<$ & $>$ & $<$ & NN, s70 & $\begin{array}{l}560780 \\
560916 \\
561000\end{array}$ & 561258 & 1 & 1 & 42 & $258-478$ & $\mathrm{n}$ & $\begin{array}{c}>, S\left(^{*}\right) \\
(560802- \\
560753)\end{array}$ \\
\hline $\mathrm{sm} / 6$ & 902 & SMc02597 & $>$ & $<$ & $>$ & NN, s70 & $\begin{array}{l}\text { I I98309, } \\
\text { I I98440, } \\
\text { I I } 98508\end{array}$ & 1198093 & 1 & 1 & 42 & $212-416$ & $\mathrm{n}$ & - \\
\hline $\mathrm{sm} / 8$ & 277 & SMc0I425 & $<$ & $>$ & $>$ & s70 & $\begin{array}{l}2270996 \\
2271068\end{array}$ & $227 \mid 224$ & 1 & 1 & 42 & $156-228$ & $n$ & - \\
\hline$s m 23$ & 292 & $\mathrm{SMc0} 1218$ & $<$ & $<$ & $>$ & NN & 1706863 & $17067 \mid 5$ & I & 3 & 34 & 148 & $n$ & - \\
\hline$s m 25$ & 396 & SMc04289 & $<$ & $>$ & $>$ & s70, PhoB & $\begin{array}{l}2210185 \\
2210260 *\end{array}$ & 2210322 & 1 & 2 & 33 & $62-137$ & $n$ & - \\
\hline$s m 26$ & 1270 & SMc04453 & $<$ & $<$ & $>$ & NN & 2321447 & 2321055 & 2 & 1 & 33 & 392 & y & $\begin{array}{c}<, A, C, O, S \\
(2321429- \\
2321478)\end{array}$ \\
\hline$s m 28$ & 826 & SMc030I4 & $>$ & $<$ & $>$ & NN, s70 & $\begin{array}{l}713461, \\
713680, \\
713763\end{array}$ & 713290 & 1 & 1 & 32 & $170-463$ & $\mathrm{n}$ & - \\
\hline $\operatorname{sm} 30$ & 644 & SMc0I 257 & $<$ & $>$ & $>$ & $\begin{array}{c}\text { NN, s70, } \\
\text { PhoB }\end{array}$ & $\begin{array}{l}1518613 \\
1518748 *\end{array}$ & 1518988 & 1 & 1 & 32 & $234-375$ & $\mathrm{n}$ & - \\
\hline
\end{tabular}

I sRNA candidates reported in contemporary studies retain their original notation (smrC\# or sra\#) [26,27]. Otherwise, the designation sm\# corresponds to the full list of sRNA predictions obtained in this work by the global scoring procedure (Additional file 17). Candidate IgRs are sorted according to its descending global score (GS). Bolded candidates have been verified experimentally $([26,27]$; this work).

${ }^{2} \mathrm{NN}$, neural network promoter prediction; s70, $\sigma^{70}$-dependent promoter; s54, RpoN $\left(\sigma^{54}\right)$-dependent promoter promoter; PhoB, putative PhoB binding site.

${ }^{3}$ The coordinates of all putative promoters and/or transcription factor binding sites within an IgR are presented. The position given for NN is the $3^{\prime}$ end of the identified sequence. For $\sigma^{70}$ the position given is 7 bases downstream of the $3^{\prime}$ end of the -10 hexamer. The $3^{\prime}$ end of the predicted binding site for $\sigma^{54}$ and PhoB is indicated with an asterisk.

${ }^{4}$ Given is the position of the last uridine at the end of the terminator sequence.

${ }^{5}$ Range of possible lengths based on putative 5 ' and 3 ' ends.

${ }^{6} \mathrm{y}$, candidate present in sRNAPredictHT search (Additional file 4); n, candidate absent in sRNAPredictHT output.

${ }^{7}$ As described in Table I. 


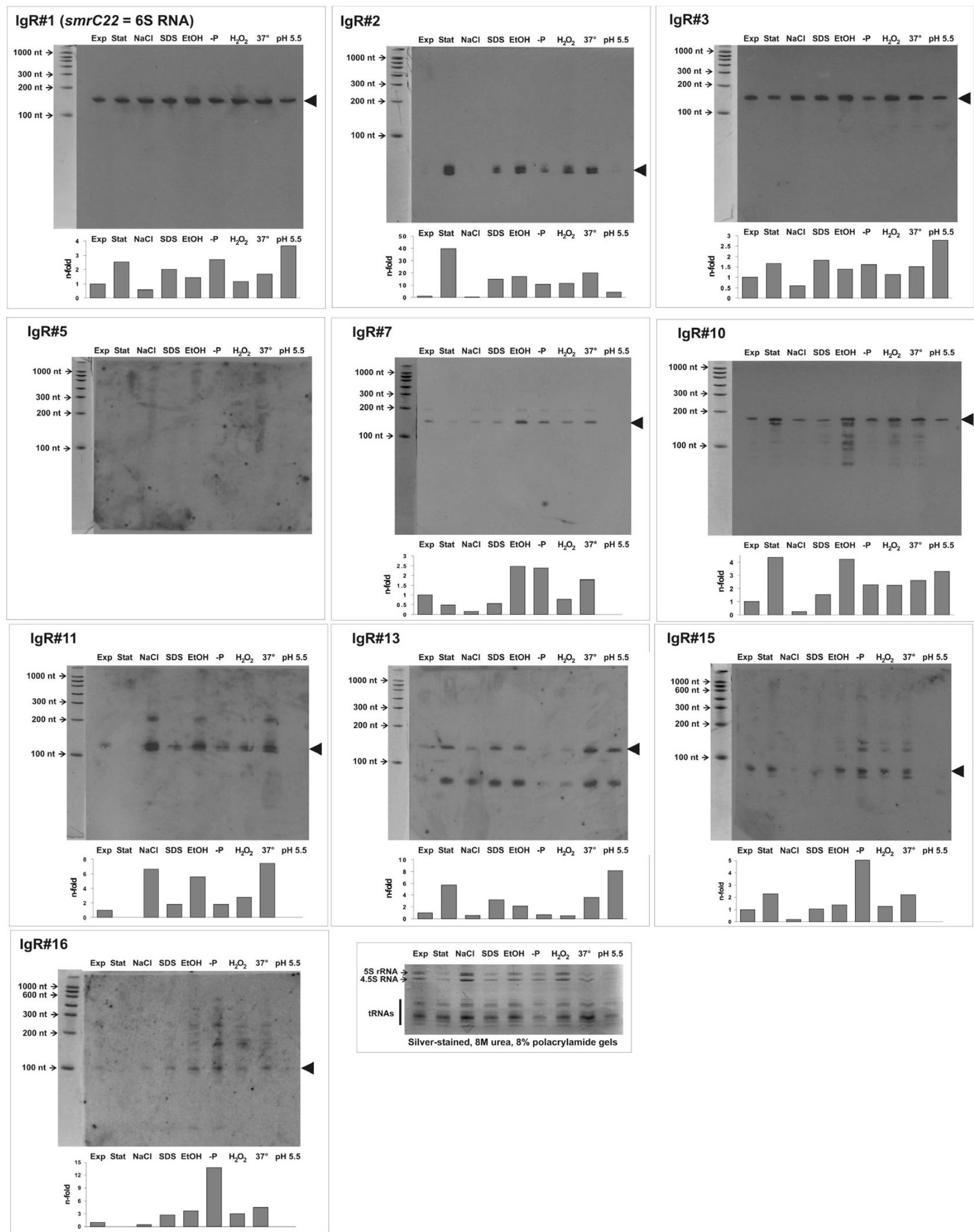

Figure I (see legend on next page) 
Figure I (see previous page)

Northern blot analysis of putative sRNAs encoded in the chromosome of S. meliloti strain 20I I. Total RNA was isolated from S. meliloti $20 \mathrm{II}$ cells grown at $28^{\circ} \mathrm{C}$ with agitation $(120 \mathrm{rpm})$ in $\mathrm{RDM}$ minimal medium and harvested at $\mathrm{OD}_{600}=$ 0.5 (Exp) or at $\mathrm{OD}_{600}=3.9$ (Stat). Total RNA was also isolated from cells subjected to high salt stress $(\mathrm{NaCl} ; 0.3 \mathrm{M} \mathrm{NaCl}$ in RDM, OD $600=0.55)$, membrane stress $\left(E t O H ;\right.$ RDM with $2 \% \mathrm{v} / \mathrm{v}$ ethanol, $\mathrm{OD}_{600}=1.6$; or SDS; RDM with $0.1 \%$ w/v SDS; $\left.\mathrm{OD}_{600}=1.0\right)$, phosphate starvation (-P; RDM with 0.1 mM phosphate and $10 \mathrm{mM}$ MOPS pH 7.0, OD $600=1.0$ ), oxidative stress $\left(\mathrm{H}_{2} \mathrm{O}_{2}\right.$; RDM with 0.I mM H $\left.\mathrm{O}_{2}, \mathrm{OD}_{600}=\mathrm{I} . \mathrm{I}\right)$, heat stress $\left(37^{\circ} ; \mathrm{RDM}\right.$ grown at $\left.37^{\circ} \mathrm{C}, \mathrm{OD}_{600}=0.95\right)$ and acid stress $(\mathrm{pH} 5.5$; treatment of exponential phase cells at $\mathrm{OD}_{600}=0.5$ during $90 \mathrm{~min}$ at $\mathrm{pH} 5.5$ before harvest). Northern hybridizations were done with PCR-generated digoxigenin-labeled dsDNA probes directed against the entire IgR or an internal fragment (see Figure 2 and Additional file 2 for further details). RNA molecular weight markers (with their sizes indicated in nt with small arrows at the left of each panel) were run with each set of RNA samples for estimation of transcript size. As exposure times were optimized for visualization here, the signal intensity does not indicate relative abundance of detected transcripts between different IgRs. Hybridization signals were quantified with Imagej software, normalized to the amount of 5S RNA, 4.5S RNA and tRNA bands detected in silver stained gels present in each sample (bottom panel) and plotted in a bar graph shown below each Northern blot. The band intensity units are relative to the normalized amount present in Exp cells, which were set as I.0.

expression of those putative sRNAs under different experimental conditions and in a different but closely related strain, so we assume that the corresponding IgRs of strain 2011 encode the sRNA homologues of 6S RNA $(s m r C 22=$ sra56), smrC9 (= sra32), sra12, sra25, smrC10 (= sra33) and smrC7 (= sra03), respectively [26,27].

To summarize, through this first compilation of putative sRNA-encoding IgRs, we obtained experimental evidence by Northern and/or microarray hybridization for eight novel $S$. meliloti RNA transcripts corresponding to candidates IgR\#2, IgR\#6, IgR\#7, IgR\#11, IgR\#15, IgR\#16, IgR\#17 and IgR\#18 (Table 1, Figure 1). Figure 2 shows the genomic context of these putative sRNA-encoding genes. The sequence alignments and associated transcriptional signals of these confirmed candidate loci are presented in Additional files 6, 7, 8, 9, 10, 11, 12, 13, 14, 15, 16 .

\section{Growth phase and stress conditions influence accumulation of detected transcripts}

For several of the sRNA transcripts detected by Northern analysis, we observed differential abundances under the various growth conditions tested (Figure 1). Transcripts originating from IgR\#1, IgR\#2, IgR\#10 and IgR\#13 were more abundant in stationary phase cells $(>2 \times)$, whereas those from IgR\#7 and IgR\#11 seemed to be downregulated in saturated cultures. The only RNA species that was clearly upregulated under high salt conditions was the one coded in IgR\#11 (>6x). Agents that alter membrane fluidity, as SDS or ethanol, induced accumulation $(>2 \times)$ of transcripts from IgR\#1, IgR\#2, IgR\#7, IgR\#10, IgR\#11 and IgR\#13. In E. coli, several sRNAs participate in the control of porin levels upon membrane stress [9]. An increase in growth temperature from 28 to $37^{\circ} \mathrm{C}$ resulted in upregulation $(>2 \times)$ of transcripts from IgR\#2 and IgR\#11. Upon phosphate starvation, the transcripts from IgR\#1, IgR\#7, IgR\#15 and IgR\#16 were upregulated. A conserved PhoB binding site [51] is not evident upstream the predicted promoter for these sRNA candidates, suggesting that the positive regulation may be indirect or PhoB-independent. Finally, exposure of S. meliloti 2011 to pH 5.5 for 90 minutes, an acid stress condition that does not support growth [60], resulted in accumulation of RNAs from IgR\#1,

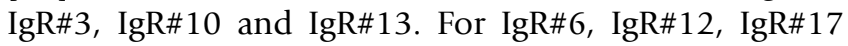
and IgR\#18, for which no Northern hybridization data was available, we could observe an enrichment of short transcripts upon 45 min of stress conditions using microarray analysis (Table 1).

The observed expression pattern for $\operatorname{IgR\# 1}$ is consistent with that observed for 6S RNA homologues in other bacteria. The transcript accumulated in stationary phase cells, in the presence of SDS, under phosphate deprivation and more markedly under conditions of acid stress. The level of 6S RNA increases along the growth curve being maximal in stationary phase in E. coli [61] and B. subtilis cells [62]. This correlates with a reduced utilization of the vegetative $\sigma^{70}$ subunit by the RNA polymerase complex in favour of alternative sigma subunits $[63,64]$. The abundance of the sRNA from IgR\#2 detected in strain 2011 was upregulated both in response to increased cell density as well as to several different stress conditions (Figure 1). This sRNA had previously been annotated as SuhB [65] but had not been subjected to experimental verification.

While the abundance of a significant number of the sRNAs identified in this study appears to be significantly affected by growth phase and/or environmental stress conditions (Figure 1, Table 1), it is still unclear how this regulation is effected. Conserved sequences suggestive of upstream regulatory sites were not detected for any of the sRNA loci confirmed in this study (Additional files 6, 7, 8, $9,10,11,12,13,14,15,16)$. Time course studies of transcript levels upon stress application together with the study of promoter expression in vitro and in planta are currently being undertaken to elucidate the regulatory mech- 
IgR\#2

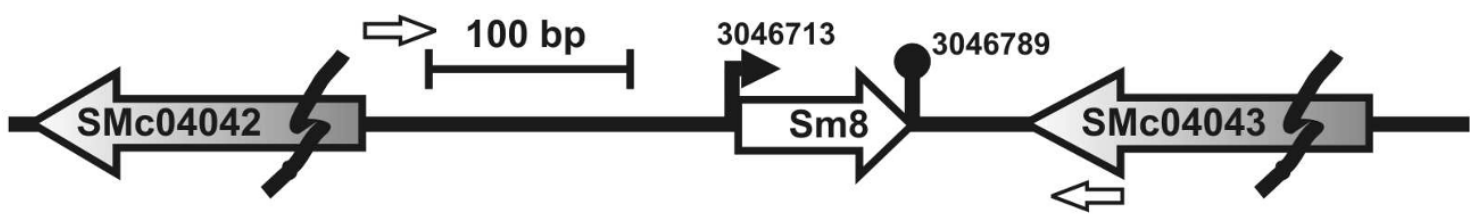

$\lg$ R\#6

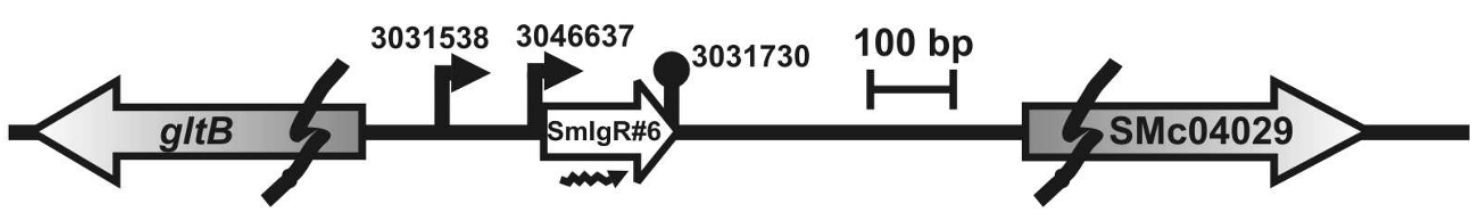

$\lg R \# 7$

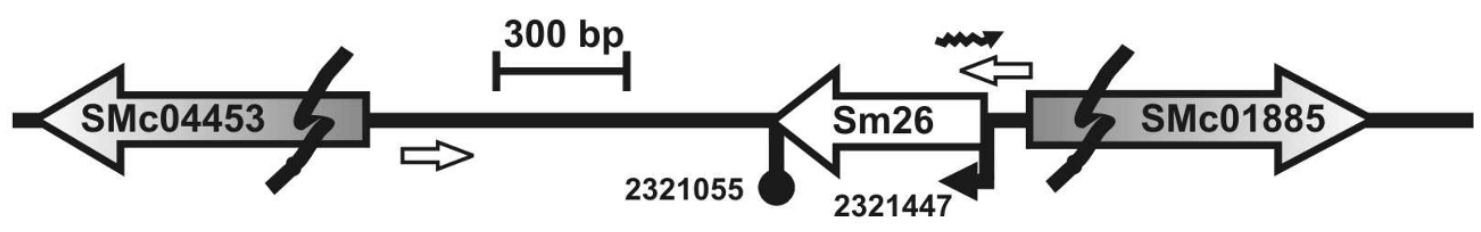

IgR\#11

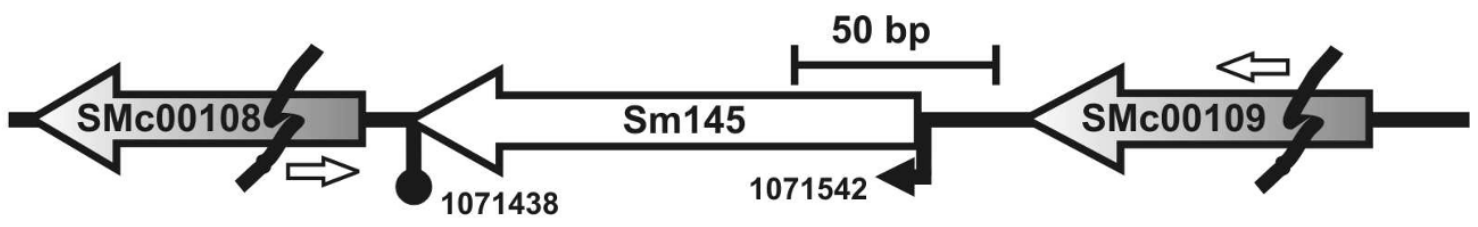

IgR\#15
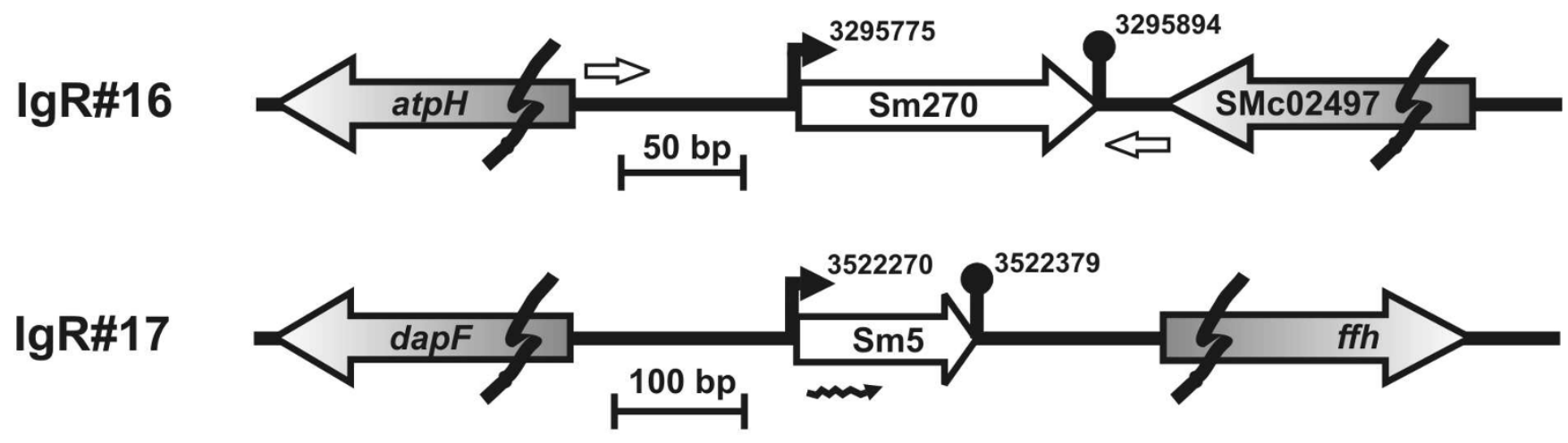

$\lg R \# 18$

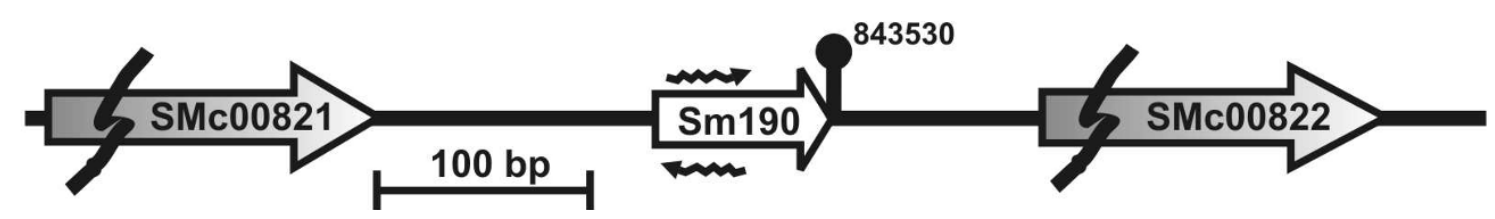

Figure 2 (see legend on next page) 
Figure 2 (see previous page)

Organization of novel S. meliloti I 02 I chromosomal loci encoding putative sRNAs with detected counterparts in S. meliloti strain $20 \mathrm{I}$ I. The IgRs encompassing novel putative sRNAs from our first compilation (Table I) are drawn to scale in the portion between breaks. The chromosomal coordinates of predicted promoters and Rho-independent terminators are indicated next to the corresponding symbols. ORFs flanking each IgR are designated with their annotated codes or gene names. sRNAs are designated according to their position in the output of global scoring for the corresponding IgR (Additional file 17) or to the corresponding IgR from Table I. Small empty arrowheads indicate the approximate position of the chromosomal target sequences for oligonucleotides used to generate probes for Northern blot. Wavy arrowheads denote the location and orientation of oligonucleotides present in SmI4kOLI microarray that detected the putative sRNA transcripts (Table I).

anisms underlying the observed differences in transcript abundance. Moreover, strains deleted for or overexpressing these sRNAs are being constructed in an effort to gain insights into the biological functions of these sRNAs both during S. meliloti growth in culture and during its symbiotic interaction with the host plant.

\section{Improvement of the bioinformatic predictive method and application to S. meliloti chromosome}

Our initial computational screen proved quite accurate in identifying both previously identified and novel sRNAs (Table 1, Figures 1 and 2). However, the parameters used in this screen were quite stringent, requiring nearly all candidate loci to be associated with a putative promoter, a predicted terminator, and conserved intergenic sequence. We therefore postulated that a significant number of $S$. meliloti sRNA-encoding genes were likely missed using our initial predictive approach. To increase the sensitivity of our computational screen, we modified our predictive algorithm so that sRNA-encoding genes are identified based on their association with any or all of the following predictive features: transcriptional terminators, promoters, primary sequence conservation, and secondary structure conservation. Bioinformatic searches using similar algorithms $[13,66]$ have often yielded a large proportion of false predictions, significantly decreasing the efficiency in which putative sRNA loci could be experimentally confirmed. Based on these previous studies, we were concerned that increasing the sensitivity of our predictive approach would result in a significant decrease in its accuracy. To address this concern, we incorporated a novel scoring algorithm that allows predicted loci to be ranked based on their likelihood of encoding a bona fide sRNAs (Figures 3 and 4). This allows stronger candidates to be readily identified and prioritized for experimental verification and characterization.

In our improved computational approach, IgRs are analysed for the presence of transcriptional signals (promoters and terminators), sequence conservation (BlastN) and secondary structure conservation (QRNA) (Figure 3), and receive a corresponding score for each item (Figure 4). Thus, prediction of a promoter and a terminator co-ori- ented but separated for $>40 \mathrm{bp}$ and $<500 \mathrm{bp}$, determines a score $=10$ for this pair of signals for a given IgR (Figure 4 ). Instead, if only one of the signals is present (terminator or promoter) or both are predicted but not co-oriented, the maximum score for each signal would be 3 (Figure 4). Similarly, a score is assigned to each IgR based on the presence of sequence and/or secondary structure conservation (see Methods). These different scoring analyses are integrated by the assignment of a global score (GS) calculated as the sum of the individual scores (Figure 4).

We first applied our improved predictive approach to the $S$. meliloti 1021 chromosome (Figure 3 illustrates the pipeline for the chromosome). We limited our searches to IgRs 150 bp or longer that do not contain annotated or nonannotated repetitive sequences. We found that $S$. meliloti IgRs containing experimentally verified sRNA transcripts were assigned a GS of 6 (IgR\#16) or higher (up to GS = 168, for the RNAse P RNA) ([26,27]; Table 3); thus we established GS $=6$ as the cut-off for sRNA prediction. Our predictive scheme identified and ranked 271 IgRs with GS $\geq 6$ (Additional file 17). We designated the candidate RNA elements as sm\# (sm1 to sm271). SsrA, RNAse P RNA, 4.5S RNA and 6S RNA were ranked within the top 32 hits (Additional file 17). All 18 of the IgRs initially selected for experimental verification (Table 1) are contained within the list of candidate sRNA genes (Additional file 17). From the entire set, we extracted a subset of 58 IgRs predicted to contain 60 transcriptional units (i.e., a predicted promoter co-oriented with a predicted terminator separated at 40-500 bp; Figure 4) (Additional file 18). Eleven of the 18 IgRs initially compiled were included in the subset of predicted transcriptional units; the other 7 IgRs were missing from this subset because either they lack typical transcription signatures (IgR\#1, IgR\#4 and IgR\#18; Table 1) or they differ significantly from the queried consensus and only became evident as conserved regions in sequence alignments with IgRs of related $\alpha$-proteobacteria (IgR\#6, IgR\#10, IgR\#12 and IgR\#16; Table 1). On the other hand, 42 of the 60 listed candidate transcriptional units in this list (Additional file 18) had not been identified previously by sRNAPredictHT (Additional file 4). 


\title{
S. meliloti 1021 chromosome (NC_003047)
}

\author{
extract ORFs, rRNAs and tRNAs \\ $2920 \lg R$ \\ extract annotated repeat elements \\ (RIMEs, Sm-repeats, Motifs AB,C) \\ retain $\operatorname{lgRs}>150$ bp \\ 1720 lgRs
}

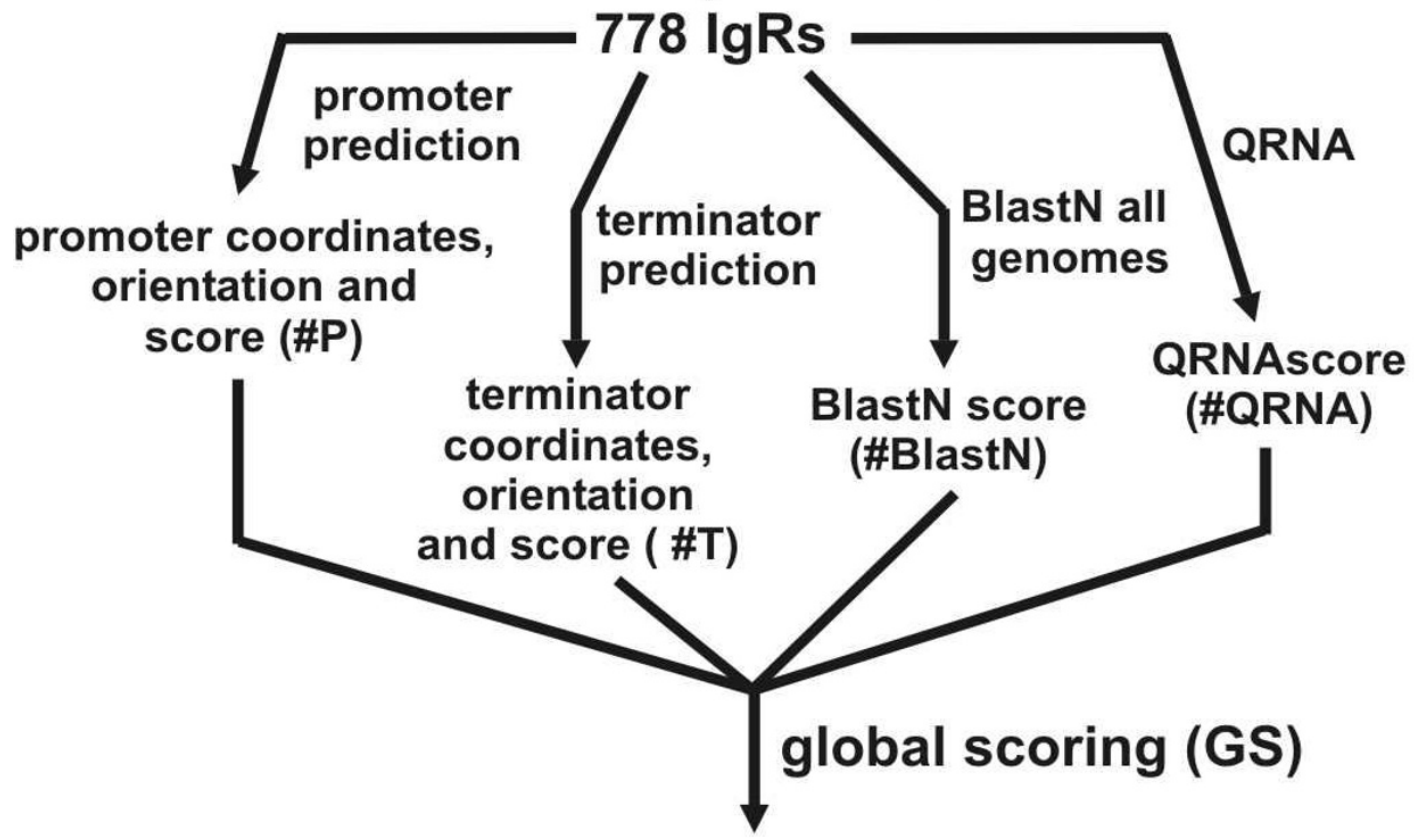

$\downarrow \begin{aligned} & \text { BlastN against itself, } \\ & \text { retain IgRs with hits } E>10^{-3}\end{aligned}$

778 IgRs 


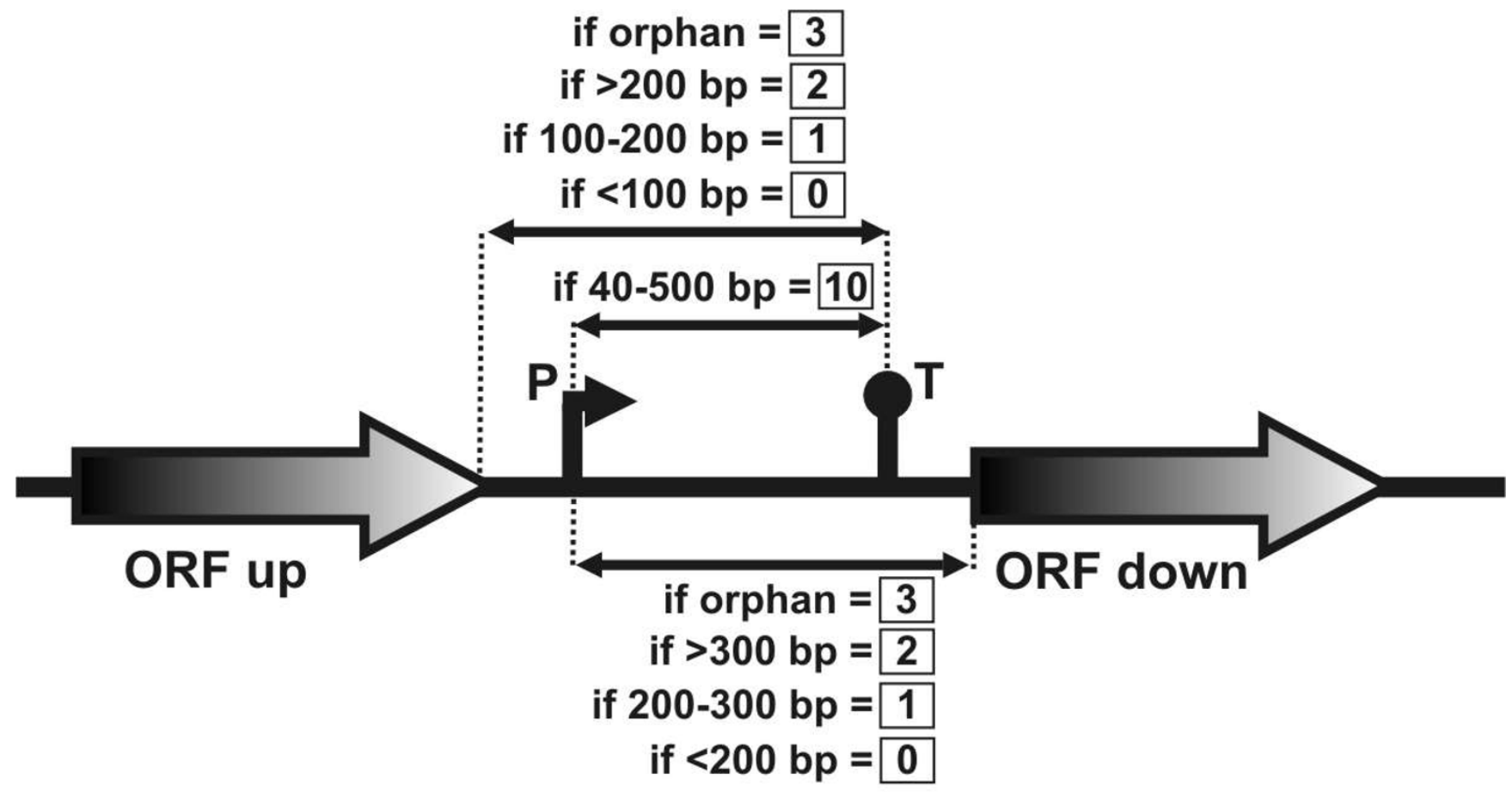

\section{If $T-P=40-500$ bp, $\quad$ GS $=10+$ \#Blast $N+$ \#QRNA}

\section{otherwise,}

\section{GS = \#P + \#T + \#BlastN +\#QRNA}

Figure 4

Summary of the scoring criteria introduced to weigh the relative position of predicted transcriptional signals in IgRs. An IgR with a co-oriented putative promoter and a terminator separated from by $40-500$ bp each other was scored I0. Every promoter-terminator pair matching the previous criterion within a single $\lg R$ was rated individually and summed to calculate the global score of that IgR. Orphan promoters were scored 3. Putative promoters were rated 2 if the 5 '-end of the co-oriented flanking CDS was farther than 300 bp, I if this distance was 200-300 bp and 0, if they were closer than 200 bp. Orphan terminators were scored 3. Predicted terminators co-oriented with flanking ORFs were scored according to their relative distance to the 3 '-end of the corresponding annotated gene, so that a score of 2 was assigned if the terminator was farther than $200 \mathrm{bp}, \mathrm{I}$ if the distance was 100-200 nt, and 0 if it was closer than $100 \mathrm{bp}$.

The 20 top-scoring candidate IgRs with predicted transcriptional units consistent with putative sRNAs are listed in Table 2. For 8 of these IgRs we observed microarray signals from exponential phase cells of strain 2011 RNA upon introduction of various stress conditions (Table 2). Thus, there is experimental evidence to date for 10 candidate sRNA loci among those top 20 IgRs (Table 2); i.e. smrC15 and $s m r C 16$ [26] (= sra41; [27]), sm4 (this work), $s m 5$ (this work), smrC14 [26] (=sm7; this work), $s m 8$ (this work), sm9 (this work), smrC9 [26] (= sra32; [27], = sm12; this work), smrC7 [26] (= sra03; [27], = sm13; this work), sra12 [27] (=sm17; this work) and $s m 26$ (this work). The high proportion of confirmed sRNAs among these highscoring loci suggests many of the 10 still unidentified candidates in this cohort correspond to bona fide sRNAencoding genes. Another remarkable feature of our predictive method is that it was able to locate quite precisely the limits of the transcriptional units. The predicted transcription start site and the last uridine of smrC15 (sm3 in Table 2 ), smrC14 (sm7 in Table 2), smrC9 (sm12 in Table 2) and smrC7 (sm13 in Table 2), differ by only 1-3 bp from the experimentally determined 5'- and 3'-termini [26]. This is also an important validation of the in silico prediction of IgR transcriptional units. It is noteworthy that the IgR with 
Table 3: Other small RNAs and cis-regulatory RNA elements detected in the chromosome of $S$. meliloti $102 \mathrm{I}$ by the global scoring procedure.

\begin{tabular}{|c|c|c|c|c|c|c|c|}
\hline Prediction & Rfam' & IgR coordinates & upstream ORF² & RNA element $^{2}$ & downstream ORF ${ }^{2}$ & GS $^{3}$ & Ref. \\
\hline RNAseP RNA & y & $2356367-2357249$ & SMc01857< & $<$ & SMc01856< & 168 & [73] \\
\hline FMN riboswitch & y & $2398059-2398373$ & SMc01608> & $>$ & ribH2 (SMc0I609)> & 127 & [67] \\
\hline 4.5S RNA (SRP) & $y$ & $259823-260123$ & SMc02904< & $>$ & dnaX> & 41 & [67] \\
\hline Glycine riboswitch & y & $1674762-1675635$ & $g c v T<$ & $<$ & SMc01242> & 37 & [67] \\
\hline ssrA (tmRNA) & $n$ & $2290955-2291 \mid 42$ & SMc0I449< & $>$ & SMc0|450> & 33 & [23] \\
\hline smrC45 (speF) & $y$ & $3105052-3105637$ & SMc02983< & $<$ & SMc02984> & 27 & [65] \\
\hline Thiamine riboswitch & y & $3532759-3533026$ & SMc $03868<$ & $>$ & SMc03869> & 19 & [67] \\
\hline ilvIH cis regulatory & $n$ & $2276530-2276876$ & SMc0I43I (ilvIH)< & $<$ & SMc0I432> & 16 & [74] \\
\hline Cobalamin riboswitch & $y$ & $1999510-1999806$ & SMc00166< & $<$ & SMc00165< & 16 & [67] \\
\hline Cobalamin riboswitch & $n$ & $2122378-2123250$ & SMc04305 (cobPW $)<$ & $<$ & SMc04306> & 14 & [67] \\
\hline Cobalamin riboswitch & $y$ & $954440-955076$ & SMc00983< & $>$ & SMc00982> & 11 & [67] \\
\hline$y b h L$ cis regulatory & y & $3505880-3506125$ & SMc03838> & $>$ & SMc03839> & 3 & [65] \\
\hline $\operatorname{ser} C$ cis regulatory & y & $2938993-2939230$ & $\operatorname{ser} C<$ & $<$ & SMc00639< & 2 & {$[65]$} \\
\hline Methionine riboswitch & y & $3461713-3461838$ & SMc03796> & $>$ & metA> & - & {$[65]$} \\
\hline Methionine riboswitch & y & $580068-580264$ & metZ< & $<$ & SMc02218> & - & [65] \\
\hline
\end{tabular}

I RNA gene or element present (y) or absent (n) in Rfam database.

2 The transcribed strand is indicated.

3 global score (GS) calculated for the element in our predictive procedure (Figure 3).

the highest global score is predicted to encode two independent sRNAs (Table 2). This region has recently been shown to encode two sRNA loci, smrC15 and smrC16 [26], with remarkably similar sizes to those predicted in this study ( $s m 3$ and $s m 3$ '). Another IgR is predicted to encode two independent sRNAs ( $s m 7$ and $s m 7$; Table 2) one of which has been experimentally verified $(\operatorname{smrC14}=s m 7)$ and the second of which awaits experimental detection [26].

Our predictive method also identified sequence elements that correspond to highly conserved small non-coding RNAs (Table 3). These predictions include the RNAseP RNA component (GS $=168)$, the SRP RNA component (4.5S RNA; GS = 41), ssrA (tmRNA; GS = 33) and 6S RNA (GS $=27)[23,27,67]$, as well as cis-regulatory RNA elements as the FMN (GS = 127), glycine ( $G S=37)$, thiamine (GS = 19), and cobalamin (GS 11-16) riboswitches and other putative cis-acting motifs as the ilvIH 5 ' trailer (GS = 16). One particular interesting locus is the $\alpha$-proteobacterial speF element $(\mathrm{GS}=27$; Table 3 ) that was first described as a possible 5'-UTR regulatory element associated with an ornithine decarboxylase mRNA gene $[65,67]$, but was recently detected as a candidate sRNA that may be independently transcribed (smrC45; [26]).

\section{Putative sRNA-encoding genes in S. meliloti megaplasmids}

We next applied our improved predictive approach to the S. meliloti 1021 megaplasmids pSymA and pSymB. The list of independent transcriptional units that may represent novel sRNA-encoding genes in pSymA and pSymB is pre- sented in Table 4. A significantly lower density of independent transcriptional units was identified in megaplasmids (an average of 8-9 sRNA genes per Mb) than in the chromosome (ca. 16 sRNA genes per Mb). It is noteworthy that most of the candidate transcripts were validated in our microarray screen (Table 4), strongly supporting the predictive methodology. Most of the transcripts were enriched > 5.5-fold (M-value > 2.5) in RNA from strain 2011 subjected to various stress stimuli, and two others ( $s m A 1$ and $s m B 7$ ) showed a $>4$-fold induction (M-value > 2) (Table 4).

One of the candidates identified in $\mathrm{pSymB}$ and detected in strain 2011 (smB5b; Table 4) seems to be a second copy of the strain 1021 transcript smrB35 [26] (= smB6; Table 4). Interestingly, the plasmid-encoded candidate $s m B 5 b$ that lies in an IgR only $3 \mathrm{~kb}$ upstream smrB35 was found to share $64 \%$ sequence identity with smrB35 (= smB6) (Additional file 19). Finally, the pSymA candidate $s m A 4 b$ was found to share sequence similarity with both chromosomally-encoded candidates $s m r C 15$ and smrC16 [26] (60\% and 67\%, respectively; Additional file 19). The putative sRNA $s m A 4 b$ is encoded within an IgR flanked by two transposable elements, ISRm5 and ISRm25, both containing their corresponding transposase genes SMa0995 and SMa0997 (Additional file 19), suggesting $s m A 4 b$ may have been acquired through horizontal transfer. The incompatibility-related pSymA and pSymB incA sRNAs were not detected as transcriptional units because they bear atypical terminators [25], but their $\sigma^{70}$-dependent promoters were precisely predicted (data not shown). 
Table 4: Putative sRNA genes predicted as independent transcriptional units in IgRs of S. meliloti I02I megaplasmids pSymA (smA\#) and pSymB (smB\#).

\begin{tabular}{|c|c|c|c|c|c|c|c|c|c|c|}
\hline \multirow{2}{*}{$\begin{array}{c}\begin{array}{c}\text { Gene or } \\
\text { Designation' }\end{array} \\
s m A I\end{array}$} & \multirow{2}{*}{$\begin{array}{c}\begin{array}{c}\text { IgR } \\
\text { length }\end{array} \\
719\end{array}$} & \multirow{2}{*}{$\begin{array}{c}\begin{array}{c}\text { Upstream } \\
\text { ORF }\end{array} \\
\text { SMa0450 }\end{array}$} & \multicolumn{3}{|c|}{$\begin{array}{l}\text { Orientation } \\
\text { Up sRNA Dn }\end{array}$} & \multirow{2}{*}{$\begin{array}{c}\text { Promoter }^{2} \\
\text { s70 }\end{array}$} & \multirow{2}{*}{$\begin{array}{c}\begin{array}{c}\text { Predicted } \\
\text { 5'-end }^{3}\end{array} \\
241667\end{array}$} & \multirow{2}{*}{$\begin{array}{c}\begin{array}{c}\text { Predicted } \\
3^{\prime} \text {-end }\end{array} \\
241961\end{array}$} & \multirow{2}{*}{$\begin{array}{c}\begin{array}{c}\text { Length } \\
\text { (nt) }\end{array} \\
294\end{array}$} & \multirow{2}{*}{$\begin{array}{c}\begin{array}{c}\text { Microarray } \\
\text { detection }^{6}\end{array} \\
>, \mathrm{C}, \mathrm{O}(*) \\
(24|99|-24 \mid 942)\end{array}$} \\
\hline & & & $>$ & $>$ & $>$ & & & & & \\
\hline $\begin{array}{l}\operatorname{smA2a} \\
\operatorname{smA2b} \\
\operatorname{smA2c}\end{array}$ & 817 & SMa0585 & $\begin{array}{l}< \\
< \\
<\end{array}$ & $\begin{array}{l}< \\
< \\
>\end{array}$ & $\begin{array}{l}> \\
> \\
<\end{array}$ & $\begin{array}{l}\text { s54 } \\
\text { s70 } \\
\text { NN }\end{array}$ & $\begin{array}{l}313672 * \\
313772 \\
313667\end{array}$ & $\begin{array}{l}313529 \\
313529 \\
314181\end{array}$ & $\begin{array}{c}143-243 \\
444\end{array}$ & $\begin{array}{c}>, \text { A, B, C, H, S } \\
(3|42| 0-3|4| 6|3| 399|-3| 40403|4| 62- \\
3|42||3| 3784-3 \mid 3833)\end{array}$ \\
\hline $\begin{array}{l}\text { smA3a } \\
\text { smA3b }\end{array}$ & 1007 & SMa0922 & $>$ & $<$ & $<$ & $\begin{array}{l}\text { NN } \\
\text { s70 }\end{array}$ & $\begin{array}{l}512362 \\
512439\end{array}$ & 512154 & $208-285$ & $\begin{array}{c}<, A, C \\
(5|237|-5 \mid 2420)\end{array}$ \\
\hline $\begin{array}{l}\operatorname{smA4a} \\
\operatorname{smA4b}\end{array}$ & 714 & SMa0995 & $\begin{array}{l}< \\
<\end{array}$ & $\begin{array}{l}> \\
>\end{array}$ & $\begin{array}{l}> \\
>\end{array}$ & $\begin{array}{l}\text { NN } \\
\text { NN }\end{array}$ & $\begin{array}{l}552600 \\
552853\end{array}$ & 552980 & $127-280$ & $>, A, B, C, H, O, S$ \\
\hline
\end{tabular}

\begin{tabular}{|c|c|c|c|c|c|c|c|c|c|c|}
\hline smA5 & 292 & SMa 1024 & $<$ & $>$ & $>$ & PhoB & $567336 *$ & 567465 & 129 & $\begin{array}{c}>, \mathrm{H}, \mathrm{S} \\
(567397-567348)\end{array}$ \\
\hline smA6 & 624 & SMaI245 & $<$ & $<$ & $<$ & NN & 682304 & 682237 & 67 & $\begin{array}{c}<, \mathrm{O} \\
(682278-682229)\end{array}$ \\
\hline smA7 & 879 & SMa I644 & $\begin{array}{l}> \\
>\end{array}$ & $>$ & $\begin{array}{l}< \\
<\end{array}$ & $\begin{array}{c}s 70 \\
N N, s 70\end{array}$ & $\begin{array}{l}916928 \\
917145\end{array}$ & 917238 & $93-310$ & - \\
\hline smA8 & 1124 & SMa2I 65 & $>$ & $>$ & $>$ & NN & 1220442 & 1220807 & 365 & $\begin{array}{c}\text { >, A, H, O, S } \\
(1220782-122083 \mathrm{I})\end{array}$ \\
\hline$s m B I$ & 282 & SMb20203 & $>$ & $>$ & $>$ & NN & 213506 & 213617 & 111 & - \\
\hline$s m B 2$ & 495 & SMb20366 & $>$ & $>$ & $<$ & NN & 379143 & 379269 & 126 & $\begin{array}{c}>, \text { H, S } \\
(379|80-379| 3 \mid)\end{array}$ \\
\hline $\begin{array}{l}s m B 3 a \\
s m B 3 b \\
s m B 3 c\end{array}$ & 750 & SMb205I6 & $\begin{array}{l}> \\
> \\
>\end{array}$ & $\begin{array}{l}< \\
< \\
<\end{array}$ & $\begin{array}{l}< \\
< \\
<\end{array}$ & $\begin{array}{l}\text { NN } \\
\text { NN } \\
\text { s70 }\end{array}$ & $\begin{array}{l}541963 \\
541911 \\
541833\end{array}$ & 541769 & 64-194 & $\begin{array}{c}<, \mathrm{H} \\
(54|835-54| 884)\end{array}$ \\
\hline $\begin{array}{l}s m B 4 a \\
s m B 4 b\end{array}$ & 592 & SMb20543 & $<$ & $<$ & $<$ & $\begin{array}{l}\mathrm{NN}, \mathrm{s} 70 \\
\text { s70 }\end{array}$ & $\begin{array}{l}571374 \\
571340\end{array}$ & 571217 & $123-157$ & $\begin{array}{c}<, \mathrm{A}, \mathrm{C} \\
(57|27|-57|22257| 222-57|27|)\end{array}$ \\
\hline $\begin{array}{l}s m B 5 a \\
s m B 5 b\end{array}$ & 1623 & SMb20548 & $\begin{array}{l}> \\
>\end{array}$ & $>$ & $>$ & $\begin{array}{c}\text { NN } \\
N N, \text { s70 }\end{array}$ & $\begin{array}{l}574477 \\
574621\end{array}$ & 574763 & $142-286$ & $\begin{array}{c}>, A, s B, C, H, O, S \\
(574671-574622)\end{array}$ \\
\hline
\end{tabular}




\begin{tabular}{|c|c|c|c|c|c|c|c|c|c|c|}
\hline smrB35 (smB6) & 751 & SMb2055I & $<$ & $>$ & $>$ & s70 & 57773 I & 577869 & 138 & $\begin{array}{c}>, \text { O, S } \\
(577781-577732)\end{array}$ \\
\hline$s m B 7$ & 196 & SMb21316 & $<$ & $>$ & $>$ & s70 & 983786 & 983878 & 92 & $\begin{array}{c}>, S(*) \\
(983845-983796)\end{array}$ \\
\hline$s m B 8$ & 767 & SMb20872 & $<$ & $<$ & $<$ & $\begin{array}{l}\text { NN, s70 } \\
\text { s70 }\end{array}$ & $\begin{array}{l}1279964 \\
12811110\end{array}$ & I 279836 & $128-274$ & $\begin{array}{c}<, \text { A, O } \\
(1279979-1280028|280| 82-|28023|)\end{array}$ \\
\hline$s m B 9$ & 1014 & SMb20916 & $<$ & $>$ & $>$ & s70 & $1325 \mid 40$ & 1325588 & 448 & $\begin{array}{c}>, H \\
(1325459-1325508)\end{array}$ \\
\hline $\operatorname{smB} 10$ & 506 & SMb20748 & $<$ & $<$ & $>$ & NN & 1537794 & 1537673 & 121 & - \\
\hline
\end{tabular}

I sRNA candidates verified experimentally retain their original notation [26]

${ }^{2} \mathrm{NN}$, neural network promoter prediction; s70, $\sigma^{70}$-dependent promoter; s54, RpoN $\left(\sigma^{54}\right)$-dependent promoter promoter; PhoB, putative PhoB binding site.

${ }^{3}$ The coordinates of all putative promoters and/or transcription factor binding sites within an lgR are presented. The position given for NN is the $3^{\prime}$ end of the identified sequence. For $\sigma^{70}$ the

position given is 7 bases downstream of the $3^{\prime}$ end of the -10 hexamer. The $3^{\prime}$ end of the predicted binding site for $\sigma^{54}$ and PhoB is indicated with an asterisk.

${ }^{4}$ Given is the position of the last uridine at the end of the terminator sequence.

${ }^{5}$ Range of possible lengths based on putative 5 ' and 3 ' ends.

${ }^{6}$ As described in Table 1. 


\section{Comments on the global scoring procedure}

Our findings suggest that the GS method is effective in identifying both known and novel sRNA genes. Half of the chromosomal and $80 \%$ of the megaplasmid IgRs predicted to contain transcriptional units suggestive of sRNA genes have been validated experimentally in this (Tables 2 and 4$)$ and other works $[26,27]$. However, it is yet unclear what proportion of all candidate predictions correspond to false positives. On the other hand, the number of potential sRNA loci may be underestimated in this study as we have ignored protein coding DNA sequences as a source of sRNA transcripts $[56,57,68]$. Another underestimation comes from the possibility that certain sRNA genes may require RNA polymerase sigma factors different from those screened here or may have atypical terminator sequences (as the aforementioned incA genes from pSymA and pSymB). As consensus sequences for additional $S$. meliloti transcription factors are determined, the modular design of our predictive protocol will allow these motifs to be readily incorporated into future searches. It is important to note that our method does not exclude the possibility that the identified putative sRNA loci, if expressed, do translate into short peptides [69] or are integral parts of mRNAs such as 5'-UTR leader regions. In fact, there are reports of sRNAs that are generated by post-transcriptional processing of mRNAs [70] including self-cleavage of riboswitch elements [71]. Our systematic procedure for IgR sorting is largely dependent on the utilization of open source tools (e.g., TranstermHP, Fuzznuc, Rsat, NNPP, BlastN, Rfam, QRNA) Thus, this methodology could be readily applied to any annotated DNA sequence for which appropriate BLAST partners and promoter consensus sequences are available. One key feature of our GS methodology is that the relative weighing of individual scores for transcriptional signatures and conservation features may be modified to generate different priority listings of candidate IgRs. We therefore anticipate that our predictive approach can be flexibly implemented in identifying sRNAs in many other bacterial species.

\section{Conclusion}

We have utilized the chromosomal DNA information of the sequenced strain $S$. meliloti 1021 to compile a first list of candidate sRNA genes (Table 1). By a combination of Northern hybridization and microarray analysis of RNA from the highly similar strain 2011, we here report eight novel sRNA loci (Table 1, Figures 1 and 2). Significant variation of transcript abundance was observed for many of the confirmed sRNAs of our first compilation under different growth conditions (Figure 1 and Table 1), providing important clues into their regulation and potential regulatory function.

The experimentally verified non-coding RNAs of $S$. meliloti, other than ssrA, 4.5S RNA, 6S RNA and RNAse P
RNA ([26,27], this work), may encode regulatory sRNAs of the base-pairing mechanism. Two lines of sequencebased evidence suggest that $S$. meliloti and probably other $\alpha$-proteobacteria as well, does not encode sRNAs of the molecular mimic type. First, no homologues of the translational regulator RNA-binding proteins of the RsmA/ CsrA family could be detected by aminoacid sequence similarity (PSI-BLAST) in $\alpha$-proteobacteria. Second, when we applied the CSRNA_FIND algorithm [72] to S. meliloti 1021 chromosomal and pSym IgRs, it did not detect a significantly higher density over the average of A(R)GGA sequence motifs, the hallmark of the molecular mimic sRNAs of the RsmZ/CsrB family [8]. Thus, most likely, $S$. meliloti only contains regulatory sRNA genes of the antisense trans-acting type [4].

To identify additional S. meliloti sRNA genes, we conducted a bioinformatic screen with a novel algorithm designed to more sensitively detect previously unannotated genes. The results of these screens significantly expand the list of putative sRNA-encoding IgRs in the three replicons of $S$. meliloti 1021 and in closely related $\alpha$ proteobacteria. Importantly, microarray data provided a strong support to our GS approach for prediction of putative sRNA-encoding genes (Tables 2 and 4). One advantage of the scoring criterion used here is that allows the strongest candidate loci to be prioritized for experimental verification and characterization. Thus, as bioinformatic screens continue to identify putative sRNA-encoding genes at rates that far exceed the throughput of existing experimental tools for sRNA confirmation and characterization, this prioritization of candidate genes should be very helpful in confirming and unravelling the diverse biological roles of these important and ubiquitous riboregulators.

\section{Authors' contributions}

CV conceived of the study, coordinated the research, carried out Northern blot analysis and wrote the manuscript; JL executed the bioinformatics search with sRNApredictHT; JPS carried out microarray hybridization experiments and microarray data analysis; JR contributed to the analysis of microarray data; $\mathrm{AB}$ designed the transcriptomic experiments and produced the microarrays; GP participated in the design, programming and execution of the Global Score algorithm. All authors revised and approved the final version of the manuscript. 


\section{Additional material}

\section{Additional file 1}

Crude sRNApredictHT predictions on S. meliloti 1021 genome. Direct output of the sRNAPredictHT algorithm applied to the complete set of S. meliloti 1021 chromosomal IgRs.

Click here for file

[http://www.biomedcentral.com/content/supplementary/1471-

2164-9-416-S1.xls]

\section{Additional file 2}

Oligonucleotides used for synthesis of Northern blot probes. Oligonucleotides used to PCR amplify the IgR sequences encompassing sRNA candidates.

Click here for file

[http://www.biomedcentral.com/content/supplementary/14712164-9-416-S2.xls]

\section{Additional file 3}

Denaturing PAGE fractionation of S. meliloti 2011 total RNA. Electrophoretic pattern of S. meliloti 2011 total RNA in a denaturing polyacrylamide gel ( $8.3 \mathrm{M}$ urea, $8 \%$ acrylamide and $0.2 \%$ bisacrylamide in $1 \times$ TBE buffer; $25 \mathrm{~cm}$-long). Approximately 20-60 $\mu \mathrm{g}$ of total RNA, corresponding to all cells present in $20 \mathrm{ml}$ of RDM cultures, were loaded in each lane. The gel was stained with ethidium bromide and visualized on an UV transilluminator. Under this conditions, effective fractionation of $R N A s<600$ nt was achieved. RNA bands of varying intensity in different samples are indicated with arrowheads. Stat, stationary phase cells; log, exponential phase cells; $\mathrm{NaCl} 0.3 \mathrm{M}$, saline stress, $\mathrm{H}_{2} \mathrm{O}_{2}$, oxidative stress; pH 4.0, acid stress; SDS and EtOH, membrane stress; -P, phosphate starvation; $0^{\circ} \mathrm{C}$, cold shock; $45^{\circ} \mathrm{C}$, heat shock.

Click here for file

[http://www.biomedcentral.com/content/supplementary/1471-

2164-9-416-S3.jpeg]

\section{Additional file 4}

Curated sRNApredictHT predictions. The sRNAPredictHT output listed in Additional file 1 was curated upon elimination of IgRs containing annotated and non annotated repeats.

Click here for file

[http://www.biomedcentral.com/content/supplementary/14712164-9-416-S4.xls]

\section{Additional file 5}

Expression of putative sRNAs in IgR\#4 and IgR\#14. Northern blot analysis of putative sRNAs encoded in IgR\#4 and IgR\#14. See legend to Figure 1 for details.

Click here for file

[http://www.biomedcentral.com/content/supplementary/14712164-9-416-S5.jpeg]

\section{Additional file 6}

Novel candidate sRNA gene $\mathrm{sm} 8$ in IgR\#2. Conservation of the novel candidate sRNA gene sm8 (IgR\#2) in $\alpha$-proteobacteria. Sequence alignment generated with ClustalW for the corresponding IgRs of S. meliloti 1021 (1021), Sinorhizobium medicae WSM419 (Smed), Agrobacterium tumenfaciens C58 (At), Rhizobium etli CFN42 (Retli) and Rhizobium leguminosarum $b v$ viciae 3841 (Rleg). The Rho-independent terminator was predicted for S. meliloti 1021 (see text) and confirmed from conserved positions in the alignment. The putative sigma 70dependent promoter (-10 and -35 hexamers) and transcription start site $(+1)$ were deduced from conserved positions in the alignment. The secondary structure presented for S. meliloti Sm8 RNA was calculated with the $M$ fold server [75] and corresponds to the predicted structure with lower free energy.

Click here for file

[http://www.biomedcentral.com/content/supplementary/1471-

2164-9-416-S6.jpeg]

\section{Additional file 7}

Novel candidate sRNA gene sm137 in IgR\#4. Conservation of the novel candidate sRNA gene $\operatorname{sm} 137$ (IgR\#4) in $\alpha$-proteobacteria. Sequence alignment generated with ClustalW for the corresponding IgRs of $\mathrm{S}$. meliloti 1021 (1021), S. medicae WSM419 (Smed), R. etli CFN42 (Retli) and R. leguminosarum $b v$ viciae 3841 (Rleg). The Rho-independent terminator was predicted for S. meliloti 1021 (see text) and confirmed from conserved positions in the alignment, but there was no prediction of a promoter in this IgR.

Click here for file

[http://www.biomedcentral.com/content/supplementary/14712164-9-416-S7.jpeg]

\section{Additional file 8}

Novel candidate sRNA gene smIgR\#6. Conservation of the novel candidate sRNA gene smIgR\#6 in $\alpha$-proteobacteria. Sequence alignment generated with ClustalW for the corresponding IgRs of S. meliloti 1021 (1021), R. etli CFN42 (Retli) and R. leguminosarum $b v$ viciae 3841 (Rleg). Two putative sigma 70-dependent promoters ( -10 and -35 hexamers), transcription start sites $(+1)$ and a single Rho-independent terminator were predicted for S. meliloti 1021 (see text). The secondary structure presented for both possible S. meliloti sRNAs from IgR\#6 were calculated with the Mfold server [75] and correspond to the predicted structures with lower free energy.

Click here for file

[http://www.biomedcentral.com/content/supplementary/14712164-9-416-S8.jpeg]

\section{Additional file 9}

Novel candidate sRNA gene sm26 in IgR\#7. Conservation of the novel candidate sRNA gene $\operatorname{sm} 26$ (IgR\#7) in $\alpha$-proteobacteria. Sequence alignment generated with ClustalW for the corresponding IgRs of $\mathrm{S}$. meliloti 1021 (1021), S. medicae WSM419 (Smed), R. etli CFN42 (Retli) and R. leguminosarum $b v$ viciae 3841 (Rleg). The putative sigma 70-dependent promoter (-10 and -35 hexamers), transcription start site $(+1)$ and Rho-independent terminator were predicted for $\mathrm{S}$. meliloti 1021 (see text). The secondary structure presented for S. meliloti Sm26 RNA was calculated with the Mfold server [75] and corresponds to the predicted structure with lower free energy.

Click here for file

[http://www.biomedcentral.com/content/supplementary/14712164-9-416-S9.jpeg] 


\section{Additional file 10 \\ Candidate SRNA gene sm64 (sra25) in IgR\#10. Conservation of the candidate sRNA gene sm64 (IgR\#10; sra25, [27]) in $\alpha$-proteobacteria. Sequence alignment generated with ClustalW for the corresponding IgRs of S. meliloti 1021 (1021), S. medicae WSM419 (Smed), R. etli CFN42 (Retli) and R. leguminosarum $b v$ viciae 3841 (Rleg). The putative sigma 70-dependent promoter (-10 and -35 hexamers), tran- scription start site $(+1)$ and Rho-independent terminator were predicted for $\mathrm{S}$. meliloti 1021 (see text) and confirmed from conserved positions in the alignment. The secondary structure presented for S. meliloti Sm64 RNA was calculated with the Mfold server [75] and corresponds to the predicted structure with lower free energy. \\ Click here for file \\ [http://www.biomedcentral.com/content/supplementary/1471- 2164-9-416-S10.jpeg]}

\section{Additional file 11}

Novel candidate SRNA gene sm145 in IgR\#11. Conservation of the novel candidate sRNA gene sm145 (IgR\#11) in $\alpha$-proteobacteria. Sequence alignment generated with ClustalW for the corresponding IgRs of S. meliloti 1021 (1021), S. medicae WSM419 (Smed), A. tumenfaciens C58 (At), R. etli CFN42 (Retli) and R. leguminosarum $b v$ viciae 3841 (Rleg). The putative sigma 70-dependent promoter (-10 and -35 hexamers), transcription start site $(+1)$ and Rho-independent terminator were predicted for S. meliloti 1021 (see text) and confirmed from conserved positions in the alignment. The secondary structure presented for S. meliloti Sm145 RNA was calculated with the Mfold server [75] and corresponds to the predicted structure with lower free energy. Click here for file [http://www.biomedcentral.com/content/supplementary/14712164-9-416-S11.jpeg]

\section{Additional file 12}

Novel candidate sRNA gene sm76 in IgR\#14. Conservation of the novel candidate sRNA gene sm76 (IgR\#14) in $\alpha$-proteobacteria. Sequence alignment generated with ClustalW for the corresponding IgRs of S. meliloti 1021 (1021), S. medicae WSM419 (Smed), A. tumenfaciens C58 (At), R. etli CFN42 (Retli), R. leguminosarum $b v$ viciae 3841 (Rleg), Mesorhizobium loti MAFF303099 (Mloti), Ochrobactrum anthropi ATCC49188 (Oa) and Brucella ovis ATCC25840 (Bo). The putative sigma 70-dependent promoter (-10 and -35 hexamers), transcription start site $(+1)$ and Rho-independent terminator were predicted for S. meliloti 1021 (see text) and confirmed from conserved positions in the alignment. A second putative promoter was predicted for $\mathrm{S}$. meliloti upstream then conserved one, but it seems to be specific for Sinorhizobium. The alternative secondary structures presented for $\mathrm{S}$. meliloti Sm76 RNA were calculated with the Mfold server [75] and corresponds to the predicted structure with lower free energy for the two possible transcripts.

Click here for file

[http://www.biomedcentral.com/content/supplementary/1471-

2164-9-416-S12.jpeg]

\section{Additional file 13}

Novel candidate sRNA gene $\mathrm{sm84}$ in IgR\#15. Conservation of the novel candidate sRNA gene sm84 (IgR\# 15) in $\alpha$-proteobacteria. Sequence alignment generated with ClustalW for the corresponding IgRs of $\mathrm{S}$. meliloti 1021 (1021), S. medicae WSM419 (Smed), A. tumenfaciens C58 (At), R. etli CFN42 (Retli) and R. leguminosarum $b v$ viciae 3841 (Rleg). The putative sigma 70-dependent promoter (-10 and -35 hexamers), transcription start site $(+1)$ and Rho-independent terminator were predicted for S. meliloti 1021 (see text) and confirmed from conserved positions in the alignment. The secondary structure presented for $\mathrm{S}$. meliloti Sm84 RNA was calculated with the Mfold server [75] and corresponds to the predicted structure with lower free energy.

Click here for file

[http://www.biomedcentral.com/content/supplementary/14712164-9-416-S13.jpeg]

\section{Additional file 14}

Novel candidate sRNA gene $\mathrm{sm} 270$ in IgR\#16. Conservation of the novel candidate sRNA gene sm270 (IgR\#16) in $\alpha$-proteobacteria. Sequence alignment generated with ClustalW for the corresponding IgRs of S. meliloti 1021 (1021), S. medicae WSM419 (Smed), A. tumenfaciens C58 (At), R. etli CFN42 (Retli) and R. leguminosarum bv viciae 3841 (Rleg). The putative sigma 70-dependent promoter ( -10 and -35 hexamers), transcription start site $(+1)$ and the putative Rho-independent terminator were predicted for S. meliloti 1021 (see text) and confirmed from conserved positions in the alignment. The secondary structure presented for S. meliloti Sm270 RNA was calculated with the Mfold server [75] and corresponds to the predicted structure with lower free energy.

Click here for file

[http://www.biomedcentral.com/content/supplementary/14712164-9-416-S14.jpeg]

\section{Additional file 15}

Novel candidate sRNA gene sm5 in IgR\#17. Conservation of the novel candidate sRNA gene sm5 (IgR\#17) in $\alpha$-proteobacteria. Sequence alignment generated with ClustalW for the corresponding IgRs of $\mathrm{S}$. meliloti 1021 (1021), R. etli CFN42 (Retli) and R. leguminosarum $b v$ viciae 3841 (Rleg). The putative sigma 70-dependent promoter $(-10$ and -35 hexamers), transcription start site $(+1)$ and the putative Rhoindependent terminator were predicted for $\mathrm{S}$. meliloti 1021 (see text) and confirmed from conserved positions in the alignment. The secondary structure presented for S. meliloti Sm5 RNA was calculated with the $M$ fold server [75] and corresponds to the predicted structure with lower free energy.

Click here for file

[http://www.biomedcentral.com/content/supplementary/14712164-9-416-S15.jpeg] 


\begin{abstract}
Additional file 16
Novel candidate sRNA gene sm190 in IgR\#18. Conservation of the novel candidate sRNA gene sm190 (IgR\#18) in $\alpha$-proteobacteria. Sequence alignment generated with ClustalW for the corresponding IgRs of S. meliloti 1021 (1021), R. etli CFN42 (Retli) and R. leguminosarum $b v$ viciae 3841 (Rleg). The putative Rho-independent terminator was predicted for S. meliloti 1021 (see text) and confirmed from conserved positions in the alignment. The secondary structure presented for $\mathrm{S}$. meliloti Sm190 RNA was calculated with the Mfold server [75] and corresponds to the predicted structure with lower free energy assuming that the sRNA extends along the conserved sequence upstream the terminator and includes the terminator itself.

Click here for file

[http://www.biomedcentral.com/content/supplementary/14712164-9-416-S16.jpeg]
\end{abstract}

\section{Additional file 17}

List of S. meliloti sRNA gene predictions based on the GS method. Complete list of S. meliloti 1021 chromosomal IgRs predicted to encode $s R N A s$ according to the global scoring procedure (summarized in Figure 3).

Click here for file

[http://www.biomedcentral.com/content/supplementary/14712164-9-416-S17.xls]

\section{Additional file 18}

Predicted transcriptional units in S. meliloti 1021 chromosomal IgRs. sRNA candidates identified as putative transcriptional units in intergenic regions of S. meliloti 1021 chromosome.

Click here for file

[http://www.biomedcentral.com/content/supplementary/14712164-9-416-S18.doc]

\section{Additional file 19}

Homologs of sRNA genes smrC14-smrC15 and smrB35 are present in pSymA and pSymB IgRs. Identification of extra copies of sRNA genes smrC14-smrC15 and smrB35 in pSymA and pSymB IgRs. Genetic surroundings and sequence alignments generated with ClustalW for the sRNA genes of S. meliloti $1021 \mathrm{smrC14}, \mathrm{smrC15}$ [26] and the corresponding identified homolog in pSymA (smA4b; Table 4), and for the sRNA gene smrB35 [26] and the corresponding identified homolog in pSymB (smB5b; Table 4). The putative sigma 70-dependent promoters, transcription start sites $(+1)$ and Rho-independent terminators were predicted for S. meliloti 1021 pSymA and pSymB (Table 4) and confirmed from conserved positions in the alignment.

Click here for file

[http://www.biomedcentral.com/content/supplementary/1471-

2164-9-416-S19.jpeg]

\section{Acknowledgements}

This work was supported by grants from ANPCyT (PICT 25396), CONICET (PIP 58I2) and UNQ (PPUNQ 0340/03, PUNQ 0395/07) to CV and from Deutsche Forschungsgemeinschaft (SPPI258) to AB. We thank Svenja Daschkey for her contribution to the microarray heat shock experiments, and Stephan Heeb, Dieter Haas and two anonymous reviewers for their valuable comments that contributed to enrich the manuscript. $\mathrm{CV}$ and GP are members of CONICET. All authors read and approved the final manuscript.

\section{References}

I. Storz G, Haas D: A guide to small RNAs in microorganisms. Curr Opin Microbiol 2007, I0(2):93-95.

2. Gottesman S: Micros for microbes: non-coding regulatory RNAs in bacteria. Trends Genet 2005, 2 I(7):399-404.

3. Chapman EJ, Carrington JC: Specialization and evolution of endogenous small RNA pathways. Nat Rev Genet 2007, 8(I I ):884-896.

4. Aiba $\mathrm{H}$ : Mechanism of RNA silencing by Hfq-binding small RNAs. Curr Opin Microbiol 2007, I 0(2): I34-139.

5. Prevost K, Salvail H, Desnoyers G, Jacques JF, Phaneuf E, Masse E: The small RNA RyhB activates the translation of shiA mRNA encoding a permease of shikimate, a compound involved in siderophore synthesis. Mol Microbiol 2007, 64(5): $1260-1273$

6. Masse E, Escorcia FE, Gottesman S: Coupled degradation of a small regulatory RNA and its mRNA targets in Escherichia coli. Genes Dev 2003, I7(19):2374-2383.

7. Brennan RG, Link TM: Hfq structure, function and ligand binding. Curr Opin Microbiol 2007, I 0(2): I25-133.

8. Babitzke $P$, Romeo $T$ : CsrB sRNA family: sequestration of RNA-binding regulatory proteins. Curr Opin Microbiol 2007, I0(2):156-163.

9. Valverde C, Haas D: Small RNAs controlled by two component systems. Adv Exp Med Biol 2008, 631 1:54-79.

10. Vogel J, Sharma CM: How to find small non-coding RNAs in bacteria. Biol Chem 2005, 386( ( 2): $1219-1238$.

II. Livny J, Waldor MK: Identification of small RNAs in diverse bacterial species. Curr Opin Microbiol 2007, I 0(2):96-I0I.

12. Argaman L, Hershberg R, Vogel J, Bejerano G, Wagner EG, Margalit $\mathrm{H}$, Altuvia S: Novel small RNA-encoding genes in the intergenic regions of Escherichia coli. Curr Biol 200 I, I I ( I 2):94 I-950.

13. Rivas E, Klein RJ, Jones TA, Eddy SR: Computational identification of noncoding RNAs in E. coli by comparative genomics. Curr Biol 200I, I I(I7):1369-1373.

14. Wassarman KM, Repoila F, Rosenow C, Storz G, Gottesman S: Identification of novel small RNAs using comparative genomics and microarrays. Genes Dev 200I, 15(13):1637-165I.

15. Gottesman S: The small RNA regulators of Escherichia coli: roles and mechanisms*. Annu Rev Microbiol 2004, 58:303-328.

16. Storz G, Altuvia S, Wassarman KM: An abundance of RNA regulators. Annu Rev Biochem 2005, 74:199-217.

17. Biondi EG, Pilli E, Giuntini E, Roumiantseva ML, Andronov EE, Onichtchouk OP, Kurchak ON, Simarov BV, Dzyubenko NI, Mengoni A, Bazzicalupo M: Genetic relationship of Sinorhizobium meliloti and Sinorhizobium medicae strains isolated from Caucasian region. FEMS Microbiol Lett 2003, 220(2):207-2I3.

18. Gage DJ: Infection and invasion of roots by symbiotic, nitrogen-fixing rhizobia during nodulation of temperate legumes. Microbiol Mol Biol Rev 2004, 68(2):280-300.

19. Galibert F, Finan TM, Long SR, Puhler A, Abola P, Ampe F, BarloyHubler F, Barnett MJ, Becker A, Boistard P, Bothe G, Boutry M, Bowser L, Buhrmester J, Cadieu E, Capela D, Chain P, Cowie A, Davis RW, Dreano S, Federspiel NA, Fisher RF, Gloux S, Godrie T, Goffeau A, Golding B, Gouzy J, Gurjal M, Hernandez-Lucas I, Hong A, Huizar L, Hyman RW, Jones T, Kahn D, Kahn ML, Kalman S, Keating DH, Kiss E, Komp C, Lelaure V, Masuy D, Palm C, Peck MC, Pohl TM, Portetelle D, Purnelle B, Ramsperger U, Surzycki R, Thebault $P$, Vandenbol M, Vorholter FJ, Weidner S, Wells DH, Wong K, Yeh KC, Batut J: The composite genome of the legume symbiont Sinorhizobium meliloti. Science 200I, 293(5530):668-672.

20. Barnett MJ, Fisher RF, Jones T, Komp C, Abola AP, Barloy-Hubler F, Bowser L, Capela D, Galibert F, Gouzy J, Gurjal M, Hong A, Huizar L, Hyman RW, Kahn D, Kahn ML, Kalman S, Keating DH, Palm C, Peck MC, Surzycki R, Wells DH, Yeh KC, Davis RW, Federspiel NA, Long SR: Nucleotide sequence and predicted functions of the entire Sinorhizobium meliloti pSymA megaplasmid. Proc Natl Acad Sci USA 200I, 98(I 7):9883-9888.

21. Finan TM, Weidner S, Wong K, Buhrmester J, Chain P, Vorholter FJ, Hernandez-Lucas I, Becker A, Cowie A, Gouzy J, Golding B, Puhler A: The complete sequence of the $1,683-\mathrm{kb}$ pSym $B$ megaplasmid from the N2-fixing endosymbiont Sinorhizobium meliloti. Proc Natl Acad Sci USA 200I, 98(17):9889-9894.

22. Ebeling S, Kundig $\mathrm{C}$, Hennecke $\mathrm{H}$ : Discovery of a rhizobial RNA that is essential for symbiotic root nodule development. J Bacteriol I991, I 73(20):6373-6382. 
23. Ulve VM, Cheron A, Trautwetter A, Fontenelle C, Barloy-Hubler F: Characterization and expression patterns of Sinorhizobium meliloti tmRNA (ssrA). FEMS Microbiol Lett 2007 , 269(I): II7-I23.

24. Keiler KC, Shapiro L, Williams KP: tmRNAs that encode proteolysis-inducing tags are found in all known bacterial genomes: A two-piece tmRNA functions in Caulobacter. Proc Natl Acad Sci USA 2000, 97( I4):7778-7783

25. MacLellan SR, Smallbone LA, Sibley CD, Finan TM: The expression of a novel antisense gene mediates incompatibility within the large repABC family of alpha-proteobacterial plasmids. Mol Microbiol 2005, 55(2):6 I I-623.

26. Del Val C, Rivas E, Torres-Quesada O, Toro N, Jimenez-Zurdo J: Identification of differentially expressed small non-coding RNAs in the legume endosymbiont Sinorhizobium melilot by comparative genomics. Mol Microbiol 2007, 66(5): 1080-1091.

27. Ulve VM, Sevin EW, Cheron A, Barloy-Hubler F: Identification of chromosomal alpha-proteobacterial small RNAs by comparative genome analysis and detection in Sinorhizobium meliloti strain I 02 I. BMC Genomics 2007, 8:467.

28. Sinorhizobium meliloti strain I02 I Genome Project [http:// bioinfo.genopole-toulouse.prd.fr/annotation/iANT/bacteria/rhime/]

29. Sridhar ], Rafi ZA: Identification of novel genomic islands associated with small RNAs. In Silico Biology 2007, 7:53.

30. Lung B, Zemann A, Madej MJ, Schuelke M, Techritz S, Ruf S, Bock R, Huttenhofer A: Identification of small non-coding RNAs from mitochondria and chloroplasts. Nucleic Acids Res 2006, 34( I 4):3842-3852.

31. Livny J, Brencic A, Lory S, Waldor MK: Identification of I 7 Pseudomonas aeruginosa sRNAs and prediction of sRNA-encoding genes in 10 diverse pathogens using the bioinformatic tool sRNAPredict2. Nucleic Acids Res 2006, 34(I 2):3484-3493.

32. Osteras M, Stanley J, Finan TM: Identification of Rhizobium-specific intergenic mosaic elements within an essential twocomponent regulatory system of Rhizobium species. J Bacteriol 1995, I77(19):5485-5494

33. Griffiths-Jones S, Moxon S, Marshall M, Khanna A, Eddy SR, Bateman A: Rfam: annotating non-coding RNAs in complete genomes. Nucleic Acids Res 2005:DI 2 I - 124.

34. Meade HM, Signer ER: Genetic mapping of Rhizobium meliloti. Proc Natl Acad Sci USA 1977, 74(5):2076-2078.

35. Beringer JE: $\mathbf{R}$ factor transfer in Rhizobium leguminosarum. Gen Microbiol 1974, 84(I): I88-198.

36. Vincent JM: A manual for the practical study of root nodule bacteria. Oxford: Blackwell Scientific Publications; 1970.

37. Blum H, Beier H, Gross HJ: Improved silver staining of plant proteins, RNA and DNA in polyacrylamide gels. Electrophoresis 1987, 8(2):93-99.

38. Sambrook J, Fritsch E, Maniatis T: Molecular Cloning: A Laboratory Manual. New York: Cold Spring Harbor Laboratory; 1989.

39. Abramoff MD, Magelhaes PJ, Ram S]: Image processing with ImageJ. Biophotonics International 2004, I I:36-42.

40. Zevenhuisen $L$, van Neerven A: $(1,2)-\beta-D$-glucan and acidic oligosaccharides produced by Rhizobium meliloti. Carbohydr Res 1983, I | 8: 127-134.

4I. Serrania J, Vorholter FJ, Niehaus K, Puhler A, Becker A: Identification of Xanthomonas campestris pv. campestris galactose utilization genes from transcriptome data. J Biotechnol 2008 I35(3):309-317.

42. Sinorhizobium meliloti 1021 SmI4kOLI [http:// www.cebitec.uni-bielefeld.de/transcriptomics/transcriptomics-facility/ sml4koli.html]

43. EMMA server [http://www.cebitec.uni-bielefeld.de/groups/brf/ software/emma info/]

44. Altschul SF, Gish W, Miller W, Myers EW, Lipman DJ: Basic local alignment search tool. J Mol Biol 1990, 2 I 5(3):403-4 10

45. Rivas E, Eddy SR: Noncoding RNA gene detection using comparative sequence analysis. BMC Bioinformatics 200I, 2:8.

46. Kingsford CL, Ayanbule K, Salzberg SL: Rapid, accurate, computational discovery of Rho-independent transcription terminators illuminates their relationship to DNA uptake. Genome Biol 2007, 8(2):R22.

47. Reese MG: Application of a time-delay neural network to promoter annotation in the Drosophila melanogaster genome. Comput Chem 200I, 26(I):51-56.
48. Rice P, Longden I, Bleasby A: EMBOSS: the European Molecular Biology Open Software Suite. Trends Genet 2000 , I 6(6):276-277.

49. MacLellan SR, MacLean AM, Finan TM. Promoter prediction in the rhizobia. Microbiology 2006, I 52(Pt 6): I75।-I763.

50. Dombrecht B, Marchal K, Vanderleyden J, Michiels J: Prediction and overview of the RpoN-regulon in closely related species of the Rhizobiales. Genome Biol 2002, 3( I 2):RESEARCH0076.

5I. Yuan ZC, Zaheer R, Morton R, Finan TM: Genome prediction of PhoB regulated promoters in Sinorhizobium meliloti and twelve proteobacteria. Nucleic Acids Res 2006, 34(9):2686-2697.

52. Krol E, Becker A: Global transcriptional analysis of the phosphate starvation response in Sinorhizobium meliloti strains I 02 I and 20 I I. Mol Genet Genomics 2004, 272(I): I- I7.

53. Regulatory Sequence Analysis Tools [http://rsat.ulb.ac.be/rsat/]

54. NCBI Entrez Genome. Bacteria Complete Chromosome List [http://www.ncbi.nlm.nih.gov/genomes/static/eub g.html]

55. Kaminski PA, Elmerich C: The control of Azorhizobium caulinodans nifA expression by oxygen, ammonia and by the HF-Ilike protein, NrfA. Mol Microbiol 1998, 28(3):603-6I3.

56. Axmann IM, Kensche P, Vogel J, Kohl S, Herzel H, Hess WR: Identification of cyanobacterial non-coding RNAs by comparative genome analysis. Genome Biol 2005, 6(9):R73.

57. Kawano M, Reynolds AA, Miranda-Rios J, Storz G: Detection of 5'and 3'-UTR-derived small RNAs and cis-encoded antisense RNAs in Escherichia coli. Nucleic Acids Res 2005 33(3): $1040-1050$

58. Capela D, Barloy-Hubler F, Gouzy J, Bothe G, Ampe F, Batut J, Boistard P, Becker A, Boutry M, Cadieu E, Dreano S, Gloux S, Godrie T, Goffeau A, Kahn D, Kiss E, Lelaure V, Masuy D, Pohl T, Portetelle D, Puhler A, Purnelle B, Ramsperger U, Renard C, Thebault P, Vandenbol M, Weidner S, Galibert F: Analysis of the chromosome sequence of the legume symbiont Sinorhizobium meliloti strain I 02 I. Proc Natl Acad Sci USA 2001, 98( I 7):9877-9882.

59. Wais RJ, Wells DH, Long SR: Analysis of differences between Sinorhizobium meliloti 1021 and 201 I strains using the host calcium spiking response. Mol Plant Microbe Interact 2002 I 5( I 2): I 245-1252

60. del Papa MF, Balague LJ, Sowinski SC, Wegener C, Segundo E, Abarca FM, Toro N, Niehaus K, A Ph, Aguilar OM, Martinez-Drets G, Lagares $A$ : Isolation and characterization of alfalfa-nodulating rhizobia present in acidic soils of central argentina and uruguay. Appl Environ Microbiol I999, 65(4): |420-| 427.

61. Wassarman KM, Storz G: 6S RNA regulates E. coli RNA polymerase activity. Cell 2000, I0 I(6):6 I3-623.

62. Barrick JE, Sudarsan N, Weinberg Z, Ruzzo WL, Breaker RR: 6S RNA is a widespread regulator of eubacterial RNA polymerase that resembles an open promoter. Rna 2005 I I (5):774-784.

63. Wassarman KM: 6S RNA: a small RNA regulator of transcription. Curr Opin Microbiol 2007, I 0(2): I64-I68.

64. Neusser T, Gildehaus N, Wurm R, Wagner R: Studies on the expression of 6S RNA from $E$. coli: involvement of regulators important for stress and growth adaptation. Biol Chem 2008 , 389(3):285-297.

65. Corbino KA, Barrick JE, Lim J, Welz R, Tucker BJ, Puskarz I, Manda $M$, Rudnick ND, Breaker RR: Evidence for a second class of $\mathbf{S}$ adenosylmethionine riboswitches and other regulatory RNA motifs in alpha-proteobacteria. Genome Biol 2005, 6(8):R70.

66. Chen S, Lesnik EA, Hall TA, Sampath R, Griffey RH, Ecker DJ, Blyn LB: A bioinformatics based approach to discover small RNA genes in the Escherichia coli genome. Biosystems 2002, 65(23): $157-177$

67. RNA families database [http://www.sanger.ac.uk/Software/Rfam/ ]

68. Kawano M, Storz G, Rao BS, Rosner JL, Martin RG: Detection of low-level promoter activity within open reading frame sequences of Escherichia coli. Nucleic Acids Res 2005 , 33(19):6268-6276.

69. Wadler CS, Vanderpool CK: A dual function for a bacterial smal RNA: SgrS performs base pairing-dependent regulation and encodes a functional polypeptide. Proc Natl Acad Sci USA 2007.

70. Vogel J, Bartels V, Tang TH, Churakov G, Slagter-Jager JG, Huttenhofer A, Wagner EG: RNomics in Escherichia coli detects new sRNA species and indicates parallel transcriptional output in bacteria. Nucleic Acids Res 2003, 3 I(22):6435-6443. 
7I. Barrick JE, Corbino KA, Winkler WC, Nahvi A, Mandal M, Collins J, Lee M, Roth A, Sudarsan N, Jona I, Wickiser JK, Breaker RR: New RNA motifs suggest an expanded scope for riboswitches in bacterial genetic control. Proc Natl Acad Sci USA 2004, I 0 I(I 7):642I-6426.

72. Kulkarni PR, Cui X, Williams JW, Stevens AM, Kulkarni RV: Prediction of CsrA-regulating small RNAs in bacteria and their experimental verification in Vibrio fischeri. Nucleic Acids Res 2006, 34(I I):336I-3369.

73. $\mathrm{Li}$ Y, Altman S: In search of RNase P RNA from microbial genomes. Rna 2004, I O(10): I533-1540.

74. Vitreschak AG, Lyubetskaya EV, Shirshin MA, Gelfand MS, Lyubetsky VA: Attenuation regulation of amino acid biosynthetic operons in proteobacteria: comparative genomics analysis. FEMS Microbiol Lett 2004, 234(2):357-370.

75. Zuker M: Mfold web server for nucleic acid folding and hybridization prediction. Nucleic Acids Res 2003, 3 I ( I3):3406-34 I5.

Publish with Bio Med Central and every scientist can read your work free of charge

"BioMed Central will be the most significant development for disseminating the results of biomedical research in our lifetime. "

Sir Paul Nurse, Cancer Research UK

Your research papers will be:

- available free of charge to the entire biomedical community

- peer reviewed and published immediately upon acceptance

- cited in PubMed and archived on PubMed Central

- yours - you keep the copyright

Submit your manuscript here:

http://www.biomedcentral.com/info/publishing_adv.asp
BioMedcentral 